\title{
Reaction-diffusion equations with spatially distributed hysteresis
}

\author{
Pavel Gurevich *, Roman Shamin ${ }^{\dagger}$, Sergey Tikhomirov ${ }^{\ddagger}$
}

September 8, 2018

\begin{abstract}
The paper deals with reaction-diffusion equations involving a hysteretic discontinuity in the source term, which is defined at each spatial point. In particular, such problems describe chemical reactions and biological processes in which diffusive and nondiffusive substances interact according to hysteresis law. We find sufficient conditions that guarantee the existence and uniqueness of solutions as well as their continuous dependence on initial data.
\end{abstract}

Key words. spatially distributed hysteresis, reaction-diffusion equation, well-posedness.

AMS subject classification. 35K57, 35K 45, 47J40

\section{Introduction}

The paper deals with reaction-diffusion equations involving a hysteretic discontinuity which is defined at each spatial point. In particular, these problems describe chemical reactions and biological processes in which diffusive and nondiffusive substances interact according to hysteresis law. We illustrate this by a model describing a growth of a colony of bacteria (Salmonella typhimurium) on a petri plate (see. [5,6]). Let $Q \subset \mathbb{R}^{n}$ be a bounded domain, $B(x, t)$ denote the density of nondiffusing bacteria in $Q$, while $u_{1}(x, t)$ and $u_{2}(x, t)$ denote the concentrations of diffusing buffer ( $\mathrm{pH}$ level) and histidine (nutrient) in $Q$, respectively. These three unknown functions satisfy the following equations in $Q$ :

$$
\left\{\begin{array}{l}
\frac{\partial B}{\partial t}=a v B \\
\frac{\partial u_{1}}{\partial t}=D_{1} \Delta u_{1}-a_{1} v B \\
\frac{\partial u_{2}}{\partial t}=D_{2} \Delta u_{2}-a_{2} v B
\end{array}\right.
$$

supplemented by initial and no-flux (Neumann) boundary conditions. In those equations $D_{1}, D_{2}, a, a_{1}, a_{2}>0$ are given constants. The function $v=v(x, t)$ corresponds to the growth rate of bacteria and is defined by hysteresis law. In the simplest case, $v(x, t)$ takes value 1 if $u_{1}(x, t)$ and $u_{2}(x, t)$ are large enough and value 0 if $u_{1}(x, t)$ and $u_{2}(x, t)$ are small enough. More precisely, one defines two curves $\Gamma_{\text {on }}$ and $\Gamma_{\text {off }}$ on the plane $\left(u_{1}, u_{2}\right)$, which divide the first quadrant into three parts $M_{\text {on }}, M_{\text {off }}$, and $M_{\text {on-off. Now }} v(x, t)=1$ whenever $\left(u_{1}(x, t), u_{2}(x, t)\right) \in M_{\text {on }}$ and $v(x, t)=0$ whenever $\left(u_{1}(x, t), u_{2}(x, t)\right) \in M_{\text {off }}$, while $v(x, t)$ takes either value 1 or 0 in $M_{\text {on-off }}$ depending on whether the trajectory $\left(u_{1}(x, t), u_{2}(x, t)\right)$ entered the region $M_{\text {on-off }}$ through $\Gamma_{\text {on }}$ or $\Gamma_{\text {off }}$. This hysteretic behavior is depicted at Fig. 1.1 see more details in [5, 6].

In the above example, hysteresis $v(x, t)$ may switch at different spatial points at different time moments. This allows one to divide the spatial domain $Q$ into connected subdomains: in each of these subdomains,

*Free Univerity of Berlin, Peoples' Friendship University of Russia, email: gurevichp@gmail.com

${ }^{\dagger}$ Russian Academy of Sciences, Far Eastern Branch, Institute of Marine Geology and Geophysics, email: roman@shamin.ru

${ }_{\ddagger}^{\ddagger}$ Free Univerity of Berlin, email: sergey.tikhomirov@gmail.com 
hysteresis $v(x, t)$ takes the same value ( 1 or 0 ) and thus defines spatial topology of itself. The boundaries between the subdomains are free boundaries whose motion depends both on the reaction-diffusion equations and hysteresis. The interplay between those two leads to formation of spatio-temporal patterns. First numerical simulations exhibiting such patterns have been carried out in $[5,6$, for the case where $Q$ is a disc in $\mathbb{R}^{2}$. The appearing pattern corresponds to concentric rings which are formed by $B(x, t)$ as $t \rightarrow \infty$ (see Fig. 1.2).

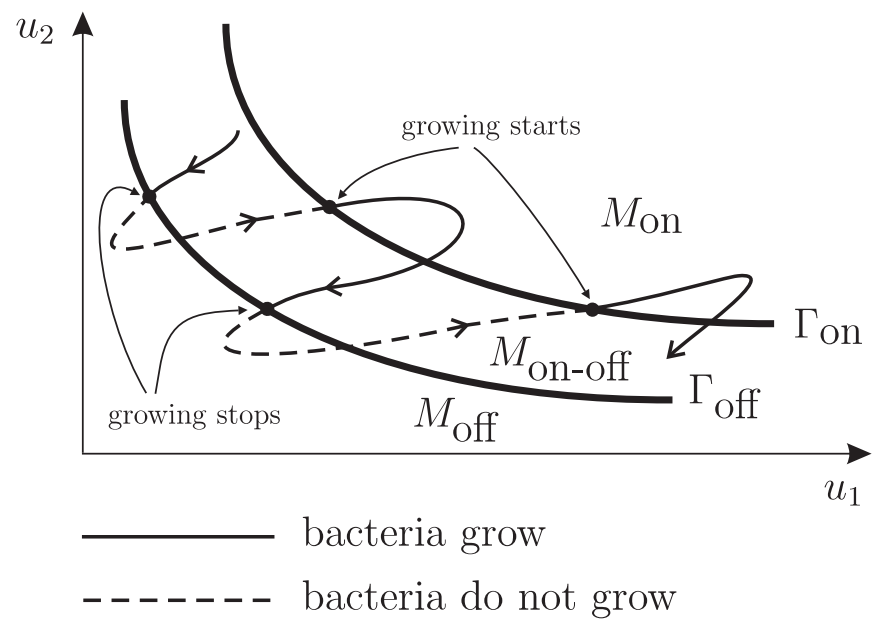

Figure 1.1: Regions of different behavior of hysteresis $\mathcal{H}$

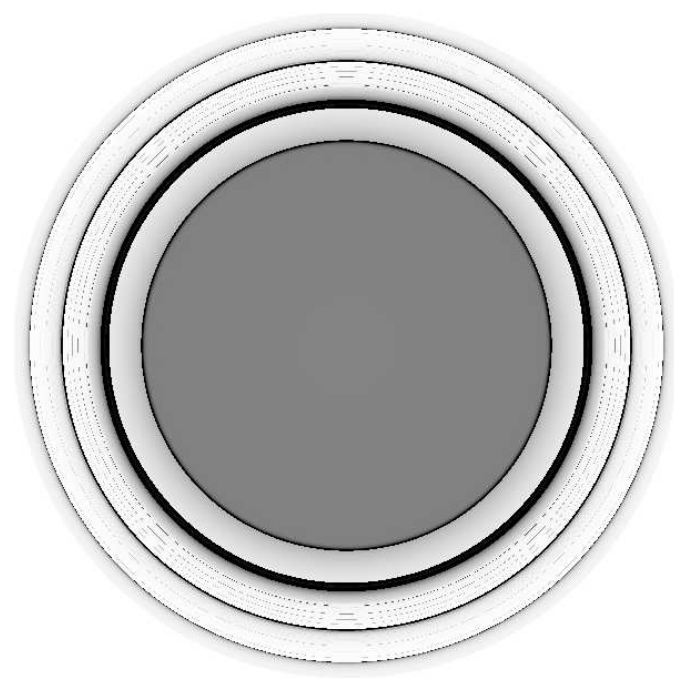

Figure 1.2: Density of bacteria after growth has stopped

First rigorous results about the existence of solutions of parabolic equations with hysteresis in the source term have been obtained in 1, 8, 16, for multi-valued hysteresis. Formal asymptotic expansions of solutions were recently obtained for some special case in [7]. Questions about the uniqueness of solutions and their continuous dependence on initial data as well as a thorough analysis of pattern formation remained open.

All the above questions are closely connected with slow-fast system where hysteresis is replaced by a "fast" function satisfying an ordinary differential equation with a small parameter at the time derivative and (typically) cubic nonlinearity. Then a possibility of singular perturbation limit is generally an open question. Some results in this direction can be found, e.g., in [3, 11, where the authors study equations of the form $u_{t}=\Delta \Phi(u)$ with cubic nonlinearity $\Phi$. However, this direction is beyond the scope of our paper.

In this paper, we obtain theorems on well-posedness of initial boundary-value problems for reactiondiffusion equations with discontinuous hysteresis in the source term. Our main assumption is the so-called spatial transversality of initial data. In the one-dimensional case, this roughly speaking means that the initial function has nonvanishing derivative at the free boundary between the above-mentioned subdomains. If this is the case, one can guarantee that a solution exists on a small time interval and can be continued as long as it remains spatially transverse. Moreover, if solution is unique it continuously depends on the initial data.

Under further natural restrictions on hysteresis, it is possible to prove that the solution is unique. For the completeness of exposition, we formulate the corresponding theorem and refer the reader to [4] for the proof.

For the clarity, all the results of the presented paper are proved for a one-dimensional domain and a scalar reaction-diffusion equation. But we note that the developed methods are not based on the maximum principle; therefore, they can be applied to systems of reaction-diffusion equations, including those in multidimensional domains (see, e.g., (1.1)).

The paper is organized as follows. In Sec. 2, we define functional spaces, introduce spatially distributed 
hysteresis (i.e., defined at every spatial point) and set the prototype problem:

$$
\left\{\begin{array}{l}
u_{t}=u_{x x}+f(u, v), \quad x \in(0,1), t>0, \\
\left.u_{x}\right|_{x=0}=\left.u_{x}\right|_{x=1}=0, \\
\left.u\right|_{t=0}=\varphi(x),
\end{array}\right.
$$

where $v(x, t)=\mathcal{H}(u(x, \cdot))(t)$ represents hysteresis at the point $x$. Loosely speaking, it is defined as follows. We fix two thresholds $\alpha$ and $\beta, \alpha<\beta$ (analogues of $\Gamma_{\text {on }}$ and $\Gamma_{\text {off }}$ in the above example). Further, we fix two functions $H_{1}(u)$ and $H_{2}(u)$. Let $x \in(0,1)$ be fixed. If $u(x, t) \leq \alpha$, then we set $v(x, t)=H_{1}(u(x, t))$; if $u(x, t) \geq \beta$, then we set $v(x, t)=H_{2}(u(x, t))$; if $\alpha<u(x, t)<\beta$, then we set $v(x, t)=H_{1}(u(x, t))$ or $H_{2}(u(x, t))$ depending on whether $u(x, t)$ entered the interval $(\alpha, \beta)$ through $\alpha$ or $\beta$, respectively (see Fig. 2.1).

Next, we discuss assumptions concerning the nonlinearity $f$, the hysteresis operator $\mathcal{H}$, and the transversality of the initial function $\varphi$. The most important notions here are the spatial topology of hysteresis and transversality. We illustrate them by the following situation. Suppose that, for a function $u(x, t)$, there exists a continuous function $b(t), t \in[0, T]$, taking values in $(0,1)$ such that

$$
\mathcal{H}(u(x, \cdot))(t)= \begin{cases}H_{1}(u(x, t)), & 0 \leq x \leq b(t), \\ H_{2}(u(x, t)), & b(t)<x \leq 1 .\end{cases}
$$

Then we say that $u$ preserves spatial topology of hysteresis in the sense that, for all $t$, there are exactly two subinterval $(0, b(t))$ and $(b(t), 1)$; hysteresis is given by $H_{1}(u)$ on one of them and by $H_{2}(u)$ on the other. In this situation, $u$ is said to be transverse on $[0, T]$ if

1. $u(x, t)<\beta$ for $x \in[0, b(t)]$,

2. $u(x, t)>\alpha$ for $x \in(b(t), 1]$

3. $u_{x}(b(t), t)>0$ whenever $u(b(t), t)=\alpha$.

In the end of Sec. 2. we formulate the main results of the paper: Theorem 2.1 (local existence of transverse solutions preserving topology), Theorem 2.2 (global existence of transverse solutions), and Theorem 2.3 and Corollary 2.1 (continuous dependence on initial data). To make the exposure complete, we also formulate Theorem 2.4 (uniqueness of transverse solutions), which is proved in [4.

We note that the failure of spatial topology may be caused by the failure of transversality but need not necessarily be (see Remark 2.2). In the latter case our methods keep working.

In Sec. 3, we collect auxiliary results. First, we recall a theorem on the well-posedness for linear parabolic problems and then for semilinear problems. We show that the hysteresis operator $\mathcal{H}$ can be treated as a continuous operator in suitable functional spaces, provided that its domain consists of spatially transverse functions (although the function $v(x, t)=\mathcal{H}(u(x, \cdot))(t)$ still may have jumps at different spatial points at different time moments). This allows us to prove Theorem 3.2, which is the main tool in the study of problem (1.2). This theorem deals with the auxiliary problem

$$
\left\{\begin{array}{l}
u_{t}=u_{x x}+f\left(u, v_{0}\right), \quad x \in(0,1), t>0, \\
\left.u_{x}\right|_{x=0}=\left.u_{x}\right|_{x=1}=0 \\
\left.u\right|_{t=0}=\varphi(x) .
\end{array}\right.
$$

The meaning of $v_{0}$ is the following. Suppose we are given functions $u_{0}(x, t)$ and $b_{0}(t)$. Then

$$
v_{0}(x, t)= \begin{cases}H_{1}\left(u_{0}(x, t)\right), & 0 \leq x \leq b_{0}(t), \\ H_{2}\left(u_{0}(x, t)\right), & b_{0}(t)<x \leq 1 .\end{cases}
$$

One can think of $b_{0}(t)$ as of a free boundary defining spatial topology of $v_{0}(x, t)$ in the sense similar to the above. However, $v_{0}$ need not coincide with hysteresis $\mathcal{H}\left(u_{0}\right)$. (It does only if $u_{0}$ preserves spatial topology and the corresponding subintervals are divided by the point $b_{0}(t)$.) 
In particular, Theorem 3.2 states that a solution $u$ of problem (1.4) exists and is unique on some time interval $[0, T]$, where $T>0$ does not depend on $u_{0}$ and $b_{0}$.

In Sec. 4, we prove the main results from Sec. 2. The local existence is proved by means of Theorem 3.2 and the Schauder fixed-point theorem for a map defined on pairs $\left(u_{0}, b_{0}\right) \in C([0,1] \times[0, T]) \times C[0, T]$. To prove global existence, we show that any local transverse solution can be continued as long as it remains transverse. The continuous dependence of solutions is also based on Theorem 3.2.

In Sec. 5, we generalize the dissipativity condition for the nonlinearities $f(u, v)$ and $H_{1}(u), H_{2}(u)$ (cf. Remark 2.3 and Example 2.1).

\section{Setting of the problem}

\subsection{Functional spaces}

Let $\Omega \subset \mathbb{R}^{n}, n \geq 2$, be a domain with piece-wise smooth boundary. We denote by $L_{q}(\Omega), q>1$, the space with the norm

$$
\|v\|_{L_{q}(\Omega)}=\left(\int_{\Omega}|v(y)|^{q} d y\right)^{1 / q} .
$$

For an integer $l \geq 0$, we denote by $C^{l}(\bar{\Omega})$ the space of functions which are continuous and have continuous derivatives up to order $l$ in $\bar{\Omega}$. For $l=0$, we write $C(\bar{\Omega})$. Denote by $C^{\gamma}(\bar{\Omega}), 0<\gamma<1$, the Hölder space with the norm

$$
\|v\|_{C^{\gamma}(\bar{\Omega})}=\|v\|_{C(\bar{\Omega})}+\sup _{x, y \in \Omega} \frac{|v(x)-v(y)|}{|x-y|^{\gamma}} .
$$

If $\Omega=(a, b)$, we will write $L_{q}(a, b), C[a, b], C^{l}[a, b]$, and $C^{\gamma}[a, b]$. If $\Omega=(0,1)$, we will write $L_{q}, C, C^{l}$, and $C^{\gamma}$, respectively. We also denote $C^{\infty}=\bigcap_{l=1}^{\infty} C^{l}$.

For a natural $l$, denote by $W_{q}^{l}=W_{q}^{l}(0,1)$ the space with the norm

$$
\|v\|_{W_{q}^{l}}=\sum_{j=0}^{l}\left\|v^{(j)}\right\|_{L_{q}}
$$

where $v^{(j)}$ is the generalized derivative of order $j$.

For any noninteger $l>0$, denote by $W_{q}^{l}=W_{q}^{l}(0,1)$ the space with the norm

$$
\|v\|_{W_{q}^{l}}=\|v\|_{W_{q}^{[l]}}+\left(\int_{0}^{1} d x \int_{0}^{1} \frac{\left|v^{([l])}(x)-v^{([l])}(y)\right|^{q}}{|x-y|^{1+q(l-[l])}} d y\right)^{1 / q}
$$

where $[l]$ is the integer part of $l$.

Let $Q_{T}=(0,1) \times(0, T)$. Denote by $C^{1,0}\left(\bar{Q}_{T}\right)$ the space of function $u(x, t)$ such that $u, u_{x} \in C\left(\bar{Q}_{T}\right)$.

We also introduce the anisotropic Sobolev space $W_{q}^{2,1}\left(Q_{T}\right)$ with the norm

$$
\|u\|_{W_{q}^{2,1}\left(Q_{T}\right)}=\left(\int_{0}^{T}\|u(\cdot, t)\|_{W_{q}^{2}}^{q} d t+\int_{0}^{T}\left\|u_{t}(\cdot, t)\right\|_{L_{q}}^{q} d t\right)^{1 / q} .
$$

The following embedding result holds (see Lemma 3.3 in [10, Chap. 2]).

Lemma 2.1. If $u \in W_{q}^{2,1}\left(Q_{T}\right)$ with $q>3$, then, for any $0 \leq \gamma<1-3 / q, u, u_{x} \in C^{\gamma}\left(\bar{Q}_{T}\right)$ and

$$
\|u\|_{C^{\gamma}\left(\bar{Q}_{T}\right)}+\left\|u_{x}\right\|_{C^{\gamma}\left(\bar{Q}_{T}\right)} \leq c\|u\|_{W_{q}^{2,1}\left(Q_{T}\right)}
$$

where $c>0$ depends on $q, \gamma$, and $T$ but does not depend on $u$. 
Throughout the paper, we fix $q$ and $\gamma$ such that

$$
q>3, \quad 0<\gamma<1-3 / q .
$$

In what follows, we will consider solutions of parabolic problems in the space $W_{q}^{2,1}\left(Q_{T}\right)$. To define a space of initial data, we will use the fact that if $u \in W_{q}^{2,1}\left(Q_{T}\right)$, then the trace $\left.u\right|_{t=t_{0}}$ is well defined and belongs to $W_{q}^{2-2 / q}$ for all $t_{0} \in[0, T]$ (see Lemma 2.4 in [10, Chap. 2]). Moreover, we can define the space $W_{q, N}^{2-2 / q}$ as the subspace of functions from $W_{q}^{2-2 / q}$ with the zero Neumann boundary conditions (it is well defined due to [14, Secs. 4.3.3 and 4.4.1]). Below we will also use the following embedding result

Lemma 2.2. If $\varphi \in W_{q}^{2-2 / q}$ with $q>3$, then, for any $0 \leq \gamma<1-3 / q, \varphi, \varphi^{\prime} \in C^{\gamma}$ and

$$
\|\varphi\|_{C^{\gamma}}+\left\|\varphi^{\prime}\right\|_{C^{\gamma}} \leq c\|u\|_{W_{q}^{2-2 / q}},
$$

where $c>0$ depends on $q$ and $\gamma$ but does not depend on $\varphi$.

\subsection{Hysteresis}

In this section, we introduce a hysteresis operator defined for functions of time variable $t$. Then we extend the definition to a spatially distributed hysteresis acting on a space of functions of time variable $t$ and space variable $x$.

We fix two numbers $\alpha$ and $\beta$ such that $\alpha<\beta$. The numbers $\alpha$ and $\beta$ will play a role of thresholds for the hysteresis operator. Next, we introduce continuous functions

$$
H_{1}:(-\infty, \beta] \mapsto \mathbb{R}, \quad H_{2}:[\alpha, \infty) \mapsto \mathbb{R} .
$$

It is convenient to extend them to $\mathbb{R}$ as follows:

$$
\begin{aligned}
& H_{1}(u)=H_{1}(\beta) \text { for } u>\beta, \\
& H_{2}(u)=H_{2}(\alpha) \text { for } u<\alpha .
\end{aligned}
$$

We assume throughout that the following condition holds.

Condition 2.1. There is a number $\sigma \in(0,1]$ such that, for any $U>0$, there exists $M=M(U)>0$ with the following property:

$$
\left|H_{j}(u)-H_{j}(\hat{u})\right| \leq M|u-\hat{u}|^{\sigma}, \quad j=1,2,
$$

whenever $|u|,|\hat{u}| \leq U$.

We fix $T>0$ and denote by $C_{r}[0, T)$ the linear space of functions which are continuous on the right in $\left[0, T\right.$ ). For any $\zeta_{0} \in\{1,2\}$ (initial configuration) and $g \in C[0, T]$ (input), we introduce the configuration function

$$
\zeta:\{1,2\} \times C[0, T] \rightarrow C_{r}[0, T), \quad \zeta(t)=\zeta\left(\zeta_{0}, g\right)(t)
$$

as follows. Let $X_{t}=\left\{t^{\prime} \in(0, t]: g\left(t^{\prime}\right)=\alpha\right.$ or $\left.\beta\right\}$. Then

$$
\zeta(0)= \begin{cases}1 & \text { if } g(0) \leq \alpha, \\ 2 & \text { if } g(0) \geq \beta, \\ \zeta_{0} & \text { if } g(0) \in(\alpha, \beta)\end{cases}
$$

and for $t \in(0, T]$

$$
\zeta(t)= \begin{cases}\zeta(0) & \text { if } X_{t}=\varnothing \\ 1 & \text { if } X_{t} \neq \varnothing \text { and } g\left(\max X_{t}\right)=\alpha \\ 2 & \text { if } X_{t} \neq \varnothing \text { and } g\left(\max X_{t}\right)=\beta\end{cases}
$$


Now we introduce the hysteresis operator (cf. [9,15])

$$
\mathcal{H}:\{1,2\} \times C[0, T] \rightarrow C_{r}[0, T)
$$

by the following rule. For any initial configuration $\zeta_{0} \in\{1,2\}$ and input $g \in C[0, T]$, the function $\mathcal{H}\left(\zeta_{0}, g\right)$ : $[0, T] \rightarrow \mathbb{R}$ (output) is given by

$$
\mathcal{H}\left(\zeta_{0}, g\right)(t)=H_{\zeta(t)}(g(t))
$$

where $\zeta(t)$ is the configuration function defined above (see Fig. 2.1).

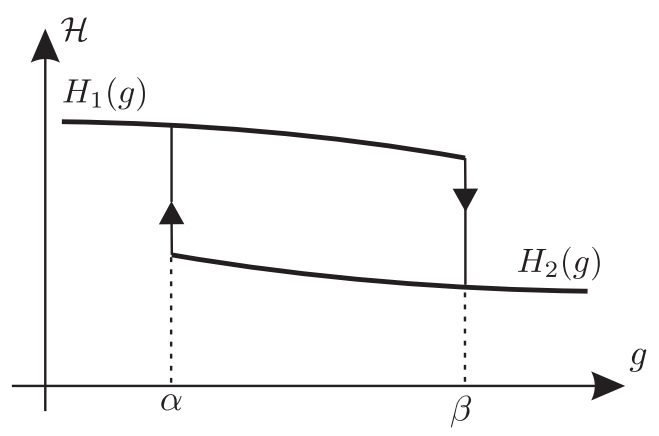

Figure 2.1: The hysteresis operator $\mathcal{H}$

Remark 2.1. One usually assumes that $H_{1}(u)-H_{2}(u)$ is sign-constant for $u \in[\alpha, \beta]$. However, we will never use this assumption in our paper.

Now we introduce a spatially distributed hysteresis. Assume that the initial configuration and the input function depend on spatial variable $x \in[0,1]$. Denote them by $\xi_{0}(x)$ and $u(x, t)$, where

$$
\xi_{0}:[0,1] \mapsto\{1,2\}, \quad u:[0,1] \times[0, T] \mapsto \mathbb{R} .
$$

Let $u(x, \cdot) \in C[0, T]$. Denote $\varphi(x)=u(x, 0)$.

Definition 2.1. We say that the function $\varphi(x)$ and the initial configuration $\xi_{0}(x)$ are consistent if, for any $x \in[0,1]$,

$$
\xi_{0}(x) \in \begin{cases}\{1\} & \text { if } \varphi(x) \leq \alpha \\ \{2\} & \text { if } \varphi(x) \geq \beta \\ \{1,2\} & \text { if } \varphi(x) \in(\alpha, \beta) .\end{cases}
$$

Assume that $\xi_{0}(x)$ and $\varphi(x)=u(x, 0)$ are consistent. Then we can define the function

$$
v(x, t)=\mathcal{H}\left(\xi_{0}(x), u(x, \cdot)\right)(t),
$$

which is called spatially distributed hysteresis.

The spatial configuration is given by

$$
\xi(x, t)=\zeta\left(\xi_{0}(x), u(x, \cdot)\right)(t) .
$$

Note that the consistency of $\xi_{0}(x)$ and $\varphi(x)$ and the fact that $\xi(x, \cdot)$ is right-continuous guarantee that

$$
\lim _{t \rightarrow 0} \xi(x, t)=\xi_{0}(x) .
$$

In this paper, we shall deal will spatially distributed hysteresis whose spatial configuration has finitely many discontinuity points in $x$ for each $t$. In particular, we assume throughout that the following holds. 
Condition 2.2. The initial configuration $\xi_{0}(x)$ has finitely many discontinuity points in $(0,1)$, i.e., there are points $0=\bar{b}_{0}<\bar{b}_{1}<\cdots<\bar{b}_{M}<\bar{b}_{M+1}=1(M \geq 1)$ such that

1. $\xi_{0}(x)=$ const for $x \in\left(\bar{b}_{i}, \bar{b}_{i+1}\right), i=0, \ldots, M$,

2. $\xi_{0}\left(\bar{b}_{i}+0\right) \neq \xi_{0}\left(\bar{b}_{i}-0\right), i=1, \ldots, M$.

Let us introduce notions of transversality and hysteresis spatial topology, which will play a central role further on.

Definition 2.2. We say that a function $\varphi \in C^{1}$ is transverse (with respect to a spatial configuration $\xi_{0}(x)$ ) if it is consistent with $\xi_{0}(x)$ and the following holds:

1. if $\varphi(\bar{x})=\alpha$ and $\varphi^{\prime}(\bar{x})=0$ for some $\bar{x} \in[0,1]$, then $\xi_{0}(\bar{x})=1$ in a neighborhood of $\bar{x}$;

2. if $\varphi(\bar{x})=\beta$ and $\varphi^{\prime}(\bar{x})=0$ for some $\bar{x} \in[0,1]$, then $\xi_{0}(\bar{x})=2$ in a neighborhood of $\bar{x}$.

Definition 2.3. We say that a function $u \in C^{1,0}\left(\bar{Q}_{T}\right)$ is transverse on $[0, T]$ (with respect to a spatial configuration $\xi(x, t))$ if, for every fixed $t \in[0, T]$, the function $u(\cdot, t)$ is transverse with respect to the spatial configuration $\xi(\cdot, t)$.

Definition 2.4. We say that a function $u \in C^{1,0}\left(\bar{Q}_{T}\right)$ preserves spatial topology (of a spatial configuration $\xi(x, t))$ on $[0, T]$ if there is $M>0$ such that, for $t \in[0, T]$, there are continuous functions

$$
0 \equiv b_{0}(t)<b_{1}(t)<\cdots<b_{M}(t)<b_{M+1}(t) \equiv 1
$$

with the properties

1. $\xi(x, t)=$ const for $x \in\left(b_{i}(t), b_{i+1}(t)\right), i=0, \ldots, M$,

2. $\xi\left(b_{i}(t)+0, t\right) \neq \xi\left(b_{i}(t)-0, t\right), i=1, \ldots, M$.

We will also say in this case that $u$ is topology preserving.

Remark 2.2. Neither of the above two definitions is equivalent to another. It may happen that the number of discontinuity points $b_{i}(t)$ in the interval $(0,1)$ decreases at some moment $t_{1}$, while $u(x, t)$ remains transverse with respect to $\xi(x, t)$ for each fixed $t \in[0, T]$. This may happen if $b_{i}(t)-b_{i+1}(t) \rightarrow 0$ as $t \rightarrow t_{1}$ for some $i \in\{0, \ldots, M\}$ (see Fig. 2.2.).
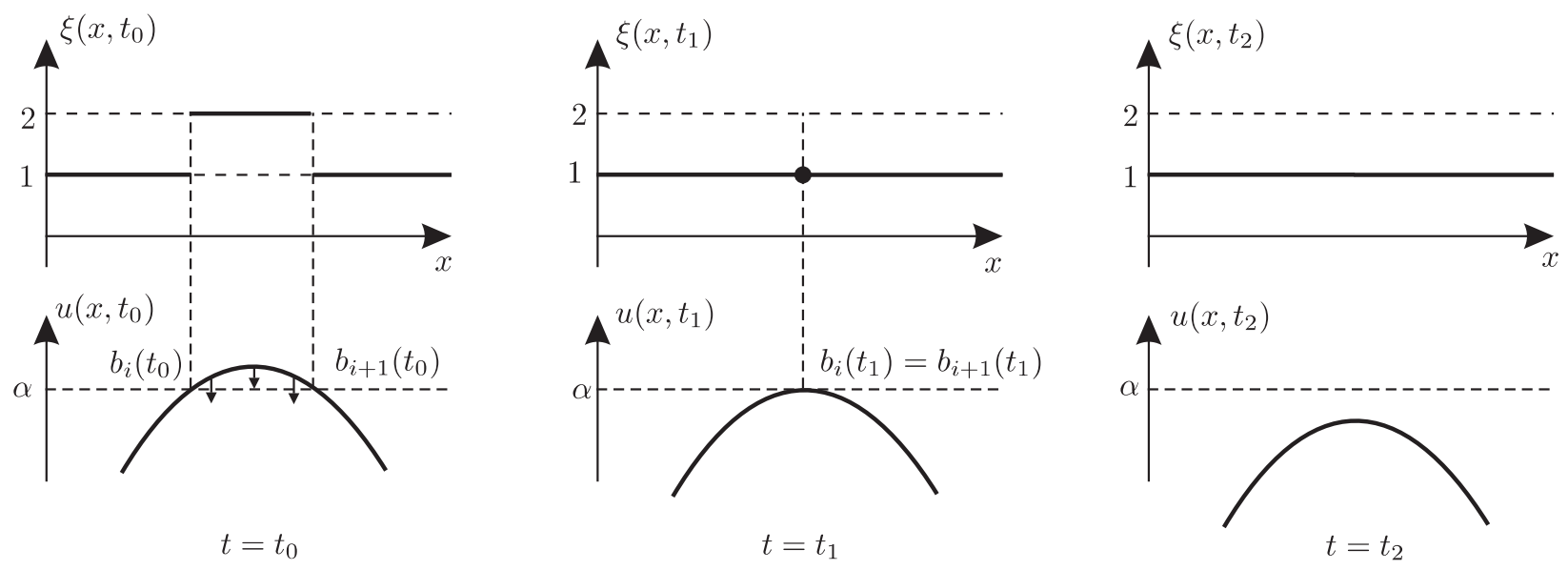

Figure 2.2: Topology changes while transversality condition holds

On the other hand, the equalities $u\left(b_{i}(t), t\right)=\alpha$ or $\beta$ and $u_{x}\left(b_{i}(t), t\right)=0$ may hold for some $t$ and some $i \in\{1, \ldots, M\}$ without changing the spatial topology (see Fig. 2.3). 

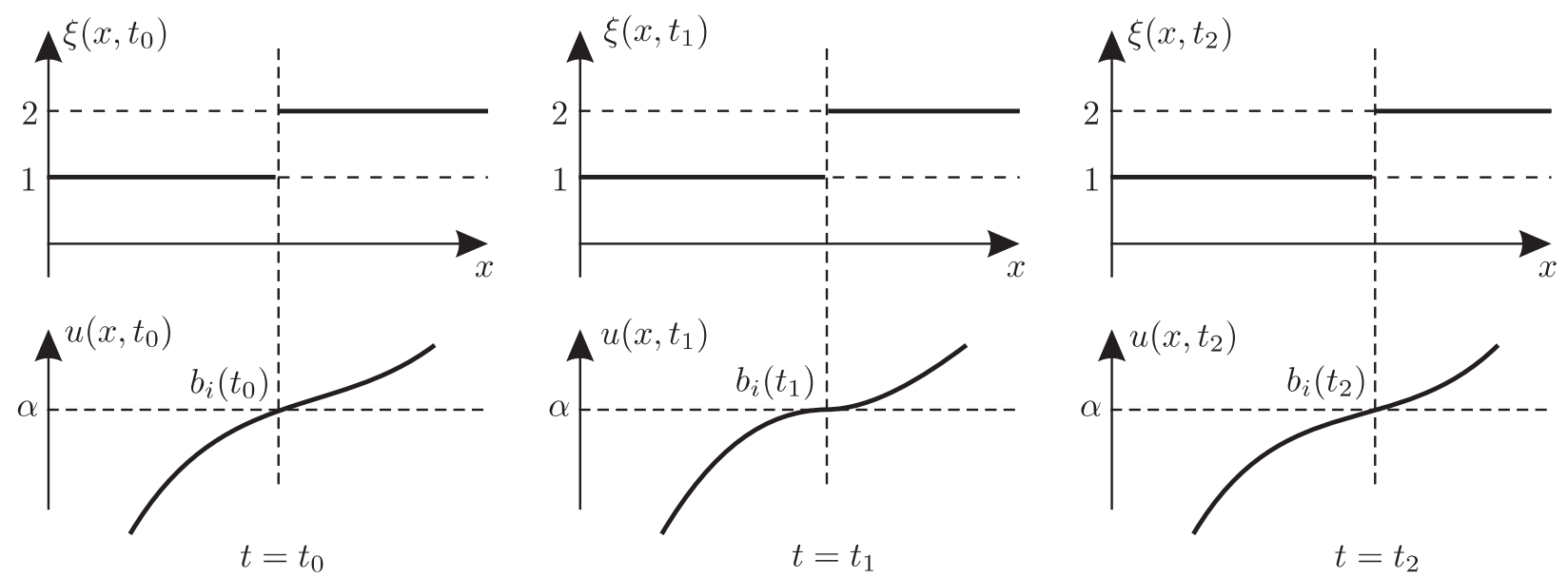

Figure 2.3: Topology preserves while transversality condition fails

\subsection{Reaction-diffusion equations with hysteresis}

The main object of this paper is the reaction-diffusion equation

$$
u_{t}=u_{x x}+f(u, v), \quad(x, t) \in Q_{T},
$$

where $v=v(x, t)$ represents the spatially distributed hysteresis given by (2.3):

$$
v(x, t)=\mathcal{H}\left(\xi_{0}(x), u(x, \cdot)\right)(t) .
$$

We also impose the Neumann boundary conditions

$$
\left.u_{x}\right|_{x=0}=\left.u_{x}\right|_{x=1}=0
$$

and the initial condition

$$
\left.u\right|_{t=0}=\varphi(x), \quad x \in(0,1) .
$$

Along with Conditions 2.1 and 2.2, we assume throughout that the right-hand side $f$, the hysteresis branches $H_{j}(u)$, and the initial data satisfy the following conditions.

Condition 2.3 (Lipschitz continuity). For any bounded set $B \subset \mathbb{R}^{2}$, there is a constant $L=L(B)>0$ such that

$$
\left|f\left(u_{1}, v_{1}\right)-f\left(u_{2}, v_{2}\right)\right| \leq L\left(\left|u_{1}-u_{2}\right|+\left|v_{1}-v_{2}\right|\right) \quad \forall\left(u_{j}, v_{j}\right) \in B, j=1,2 .
$$

Condition 2.4 (dissipativity). For any sufficiently large $U>0$ and for all $u$ such that $|u| \leq U$, we have

$$
f\left(U, H_{j}(u)\right)<0, \quad f\left(-U, H_{j}(u)\right)>0, \quad j=1,2 .
$$

Remark 2.3. In Sec. 5, we will generalize Condition 2.4 to the following:

1. for all sufficiently large $u$,

$$
f\left(u, H_{2}(u)\right) \leq 0, \quad f\left(-u, H_{1}(-u)\right) \geq 0
$$

2. there is a Lipschitz continuous function $h(u)$ such that $u h(u)>0$ for $u \neq 0$ and, for any (small) $\mu>0$, there exists $U_{\mu}>0$ such that the function

$$
f_{\mu}(u, v)=f(u, v)-\mu h(u)
$$

satisfies

$$
f_{\mu}\left(U_{\mu}, H_{j}(u)\right) \leq 0, \quad f_{\mu}\left(-U_{\mu}, H_{j}(u)\right) \geq 0 \quad \forall|u| \leq U_{\mu}, j=1,2 .
$$


Example 2.1. The simplest example of $f(u, v)$ is

$$
f(u, v)=-d u+v,
$$

where $d>0$ and

$$
\lim _{U \rightarrow+\infty}\left(-d U+\max _{|u| \leq U} H_{j}(u)\right)<0, \quad \lim _{U \rightarrow-\infty}\left(-d U+\max _{|u| \leq U} H_{j}(u)\right)>0 .
$$

The simplest example of the hysteresis in this case is given by

$$
H_{1}(u) \equiv h_{1}, \quad H_{2}(u) \equiv h_{2},
$$

where $h_{1}$ and $h_{2}$ are arbitrary real numbers without any sign restrictions (see Fig. 2.4).

Moreover, Remark 2.3 with $h(u) \equiv u$ shows that if $h_{1} \leq 0 \leq h_{2}$, then one can set $d=0$.
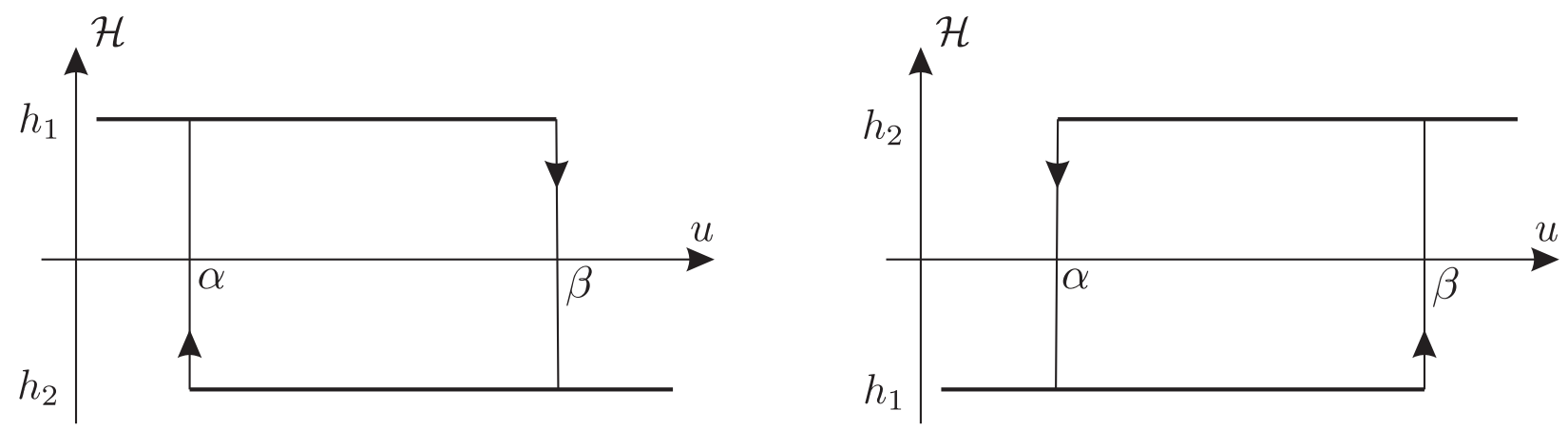

Figure 2.4: There are no sign restrictions for $h_{1}$ and $h_{2}$ in Example 2.1 both hysteresis operators are admissible, provided that $d>0$.

The natural assumption on the initial data is as follows.

Condition 2.5 (consistency). The initial spatial configuration $\xi_{0}(x)$ and the initial data $\varphi(x)$ are consistent in the sense of Definition 2.1.

To prove the existence of a solution for problem (2.6) $-(2.8)$, we will restrict a class of admissible initial data, namely we assume throughout the following.

Condition 2.6. The initial function $\varphi(x)$ is transverse in the sense of Definition 2.2 .

We give a definition of a solution of problem (2.6)-(2.8), assuming that $\varphi \in W_{q, N}^{2-2 / q}$.

Definition 2.5. A function $u(x, t)$ is called a solution of problem (2.6)-(2.8) (in $\left.Q_{T}\right)$ if $u \in W_{q}^{2,1}\left(Q_{T}\right)$, $v(x, t)$ is measurable, $u$ and $v$ satisfy equation (2.6) for a.e. $(x, t) \in Q_{T}$, and conditions (2.7) and (2.8) are satisfied in the sense of traces.

It follows from this definition and from Lemma2.1 that any solution $u(x, t)$ belongs to $C\left(\bar{Q}_{T}\right)$. Therefore, the function $v(x, t)$ is well defined by (2.3) and belongs to $L_{\infty}\left(Q_{T}\right)$.

\subsection{Main results}

Our main results are the following three theorems. In all of them, we assume that Conditions 2.1 2.6 hold and that $q$ and $\gamma$ satisfy (2.1).

Theorem 2.1 (local existence). There is a number $T>0$ such that the following holds. 
1. Any solution $u \in W_{q}^{2,1}\left(Q_{T}\right)$ of problem (2.6)-(2.8) in $Q_{T}$ is transverse and preserves spatial topology. For each $t \in[0, T]$, the function $v(\cdot, t)$ has exactly $M$ discontinuity points $b_{1}(t)<\cdots<b_{M}(t)$ in $(0,1)$. Moreover,

(a) $b_{i}(0)=\bar{b}_{i}$,

(b) $b_{i} \in C^{\gamma}[0, T]$.

2. There is at least one transverse topology preserving solution $u \in W_{q}^{2,1}\left(Q_{T}\right)$ of problem (2.6)-(2.8) in $Q_{T}$.

The next theorem deals with continuation of solutions to their maximal intervals of transverse existence.

Definition 2.6. We say that $\left[0, T_{\max }\right), T_{\max } \leq \infty$, is a maximal interval of transverse existence of a solution $u$ of problem (2.6) $-(2.8)$ if

1. for any $T<T_{\max }$, the function $u$ is a transverse solution of problem (2.6)-(2.8) in $Q_{T}$,

2. either $T_{\max }=\infty$, or $T_{\max }<\infty$ and $u$ is a solution in $Q_{T_{\max }}$, but $u\left(\cdot, T_{\max }\right)$ is not transverse with respect to $\xi\left(\cdot, T_{\max }\right)$.

Note that a solution need not be topology preserving inside its maximal interval of transverse existence (see Remark 2.2).

Theorem 2.2 (continuation). Let $u \in W_{q}^{2,1}\left(Q_{t_{0}}\right)$ be a transverse topology preserving solution of problem (2.6) -(2.8) in $Q_{t_{0}}$. Then it can be continued to a maximal interval of transverse existence $\left[0, T_{\max }\right)$, where $T_{\max }>t_{0}$ may depend on continuation.

The following theorem shows that if a transverse topology preserving solution is unique, then it continuously depends on initial function $\varphi$ and initial configuration $\xi_{0}$.

Theorem 2.3 (continuous dependence on initial data). We assume that the following hold.

1. There is a number $T>0$ such that problem (2.6)-(2.8) with initial function $\varphi \in W_{q, N}^{2-2 / q}$ and initial configuration $\xi_{0}(x)$ defined by its discontinuity points $\bar{b}_{1}<\cdots<\bar{b}_{M}$ admits a unique transverse topology preserving solution $u \in W_{q}^{2,1}\left(Q_{s}\right)$ in $Q_{s}$ for any $s \leq T$.

2. Let $\varphi_{n} \in W_{q, N}^{2-2 / q}, n=1,2, \ldots$, be a sequence of other initial functions such that $\left\|\varphi-\varphi_{n}\right\|_{W_{q, N}^{2-2 / q}} \rightarrow 0$ as $n \rightarrow \infty$.

3. Let $\xi_{0 n}(x), n=1,2, \ldots$, be a sequence of other initial configurations defined by their discontinuity points $\bar{b}_{1 n}<\cdots<\bar{b}_{M n}$ such that $\xi_{0 n}(x)=\xi_{0}(x)$ for $x \in\left(0, \min \left(b_{1}, b_{1 n}\right)\right)$ and $\bar{b}_{j n}-\bar{b}_{j} \rightarrow 0$ as $n \rightarrow \infty$, $j=1, \ldots, M$.

Then, for all sufficiently large $n$, problem (2.6)-(2.8) with initial function $\varphi_{n}$ and initial configuration $\xi_{0 n}$ has at least one transverse topology preserving solution $u_{n} \in W_{q}^{2,1}\left(Q_{T}\right)$. Each sequence of such solutions satisfies

$$
\left\|u_{n}-u\right\|_{W_{q}^{2,1}\left(Q_{T}\right)} \rightarrow 0, \quad\left\|b_{j n}-b_{j}\right\|_{C[0, T]} \rightarrow 0, \quad j=1, \ldots, M, \quad n \rightarrow \infty,
$$

where $b_{j}(t)$ and $b_{j n}(t)$ are the respective discontinuity points of the configuration functions $\xi(t)$ and $\xi_{j}(t)$, $j=1, \ldots, M$.

As we have mentioned before, a solution $u$ need not be topology preserving on its maximal interval of transverse existence $\left[0, T_{\max }\right)$. But Theorem 2.3 deals only with topology preserving solutions. The reason is the following. Let $t_{\max }\left(t_{\max }<T_{\max }\right)$ be a moment at which the solution $u$ changes topology. Although Theorem 2.3 guarantees that $u_{n}$ remain transverse and topology preserving on any interval $[0, T] \subset\left[0, t_{\max }\right)$ and approximate $u$, it may happen that each $u_{n}$ becomes nontransverse at some moment $t_{n} \in\left(T, t_{\max }\right]$ for all $n \geq N=N(T)$. This situation is illustrated by Fig. 2.5. 

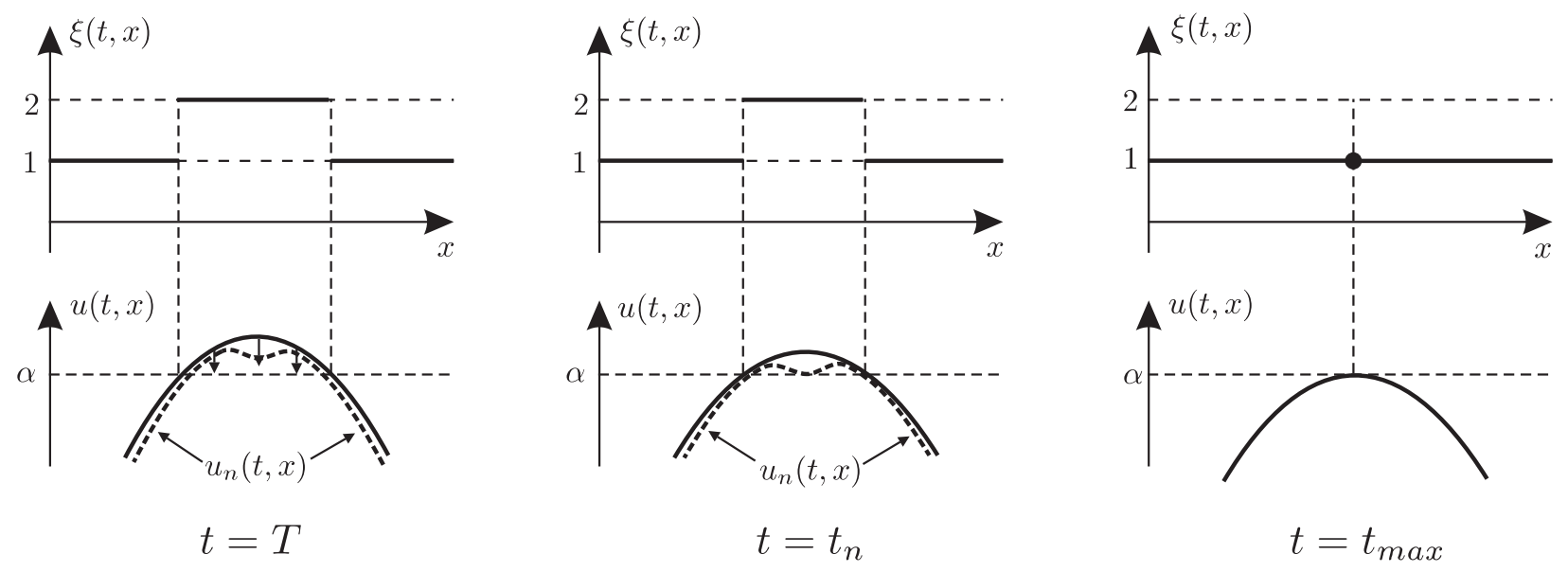

Figure 2.5: Approximation $u_{n}$ becomes nontransverse at a moment $t_{n} \in\left(T, t_{\max }\right)$

However, if we a priori know that all $u_{n}$ are transverse on some interval $[0, T] \subset\left[0, T_{\max }\right.$ ) (possibly with $T \geq t_{\max }$ ), then we can prove that $u_{n}$ approximate $u$. Now the topology of $u$ can change. This happens if some neighboring discontinuity points $b_{j}(t)$ and $b_{j+1}(t)$ of $\xi(x, t)$ converge to the same point $\hat{b}_{j}$ as $t \rightarrow t_{\max }$. Then, for $t>t_{\text {max }}$, the point $b_{j}(t)=b_{j+1}(t)=\hat{b}_{j}$ is not a discontinuity point of $\xi(x, t)$ any more. However, if we consider the functions $b_{j}(t)$ and $b_{j+1}(t)$ on the whole interval $[0, T]$ assuming them constant for $t>t_{\max }$, then we can prove that $b_{j n}(t)$ approximate $b_{j}(t)$ in $C[0, T]$. Here we use the same convention about $b_{j n}(t)$.

Let us formulate this assertion as a corollary from Theorem 2.3 .

Corollary 2.1. 1. We fix some time interval $[0, T]$, initial function $\varphi \in W_{q, N}^{2-2 / q}$, and initial configuration $\xi_{0}(x)$ defined by its discontinuity points $\bar{b}_{1}<\cdots<\bar{b}_{M}$. Assume that problem (2.6)-(2.8) with initial data $\varphi$ and $\xi_{0}(x)$ admits a unique transverse solution $u \in W_{q}^{2,1}\left(Q_{s}\right)$ in $Q_{s}$ for any $s \leq T$.

2. Let assumptions 2 and 3 of Theorem 2.3 hold. Moreover, suppose that, for each $n$, there exists a transverse solution $u_{n} \in W_{q}^{2,1}\left(Q_{T}\right)$ of problem (2.6)-(2.8) with initial data $\varphi_{n}$ and $\xi_{0 n}(x)$.

Then

$$
\left\|u_{n}-u\right\|_{W_{q}^{2,1}\left(Q_{T}\right)} \rightarrow 0, \quad\left\|b_{j n}-b_{j}\right\|_{C[0, T]} \rightarrow 0, \quad j=1, \ldots, M, \quad n \rightarrow \infty .
$$

For the completeness of exposition, we also formulate the uniqueness theorem, (see 4 ] for the proof). In the uniqueness theorem, along with Conditions 2.1 2.6 we assume that the following holds.

Condition 2.7. There is a number $\sigma \in[0,1)$ such that, for any $U>0$, there exists $M=M(U)>0$ with the properties

$$
\begin{gathered}
\left|H_{1}(u)-H_{1}(\hat{u})\right| \leq \frac{M}{(\beta-u)^{\sigma}+(\beta-\hat{u})^{\sigma}}|u-\hat{u}|, \quad \forall u, \hat{u} \in[-U, \beta), \\
\left|H_{2}(u)-H_{2}(\hat{u})\right| \leq \frac{M}{(u-\alpha)^{\sigma}+(\hat{u}-\alpha)^{\sigma}}|u-\hat{u}|, \quad \forall u, \hat{u} \in(\alpha, U] .
\end{gathered}
$$

Theorem 2.4 (uniqueness). Let the functions $H_{j}(u), j=1,2$, additionally satisfy Condition 2.7. Assume that $u, \hat{u} \in W_{q}^{2,1}\left(Q_{T_{0}}\right)$ are two transverse solutions of problem (2.6)-(2.8) in $Q_{T_{0}}$ for some $T_{0}$. Then $u=\hat{u}$.

Remark 2.4. 1 . Any locally Lipschitz continuous functions $H_{1}(u)$ and $H_{2}(u)$ satisfy Condition 2.7 Moreover, this condition covers the important case where non-Lipschitz hysteresis branches $H_{1}(u)$ and $H_{2}(u)$ appear as a singular-perturbation limit of slow-fast system (see [4).

2. On the other hand, any $H_{1}(u)$ and $H_{2}(u)$ satisfying Condition 2.1 are locally Hölder continuous with exponent $1-\sigma$ on $(-\infty, \beta]$ and $[\alpha, \infty)$, respectively. Therefore, they satisfy Condition 2.1 . 


\subsection{Technical simplification}

For the clarity of exposition, we give detailed proofs of the main results for the functions $\varphi(x)$ and $\xi_{0}(x)$ satisfying the following condition (see Fig. 2.6).

Fix some $\bar{b} \in(0,1)$.

Condition 2.8. 1. For $\bar{b} \in(0,1)$, one has

$$
\xi_{0}(x)= \begin{cases}1, & x \leq \bar{b} \\ 2, & x>\bar{b}\end{cases}
$$

2. $\varphi(x)<\beta$ for $x \in[0, \bar{b}]$.

3. $\varphi(x)>\alpha$ for $x \in(\bar{b}, 1]$.

4. If $\varphi(\bar{b})=\alpha$, then $\varphi^{\prime}(\bar{b})>0$.

It follows from this condition that the hysteresis (2.3) at the initial moment is given by

$$
\left.v\right|_{t=0}= \begin{cases}H_{1}(\varphi(x)), & x \leq \bar{b} \\ H_{2}(\varphi(x)), & x>\bar{b} .\end{cases}
$$

Clearly, Condition 2.2 (with $M=1$ and $\bar{b}_{1}=\bar{b}$ ) and Conditions 2.5 and 2.6 are satisfied in this case.

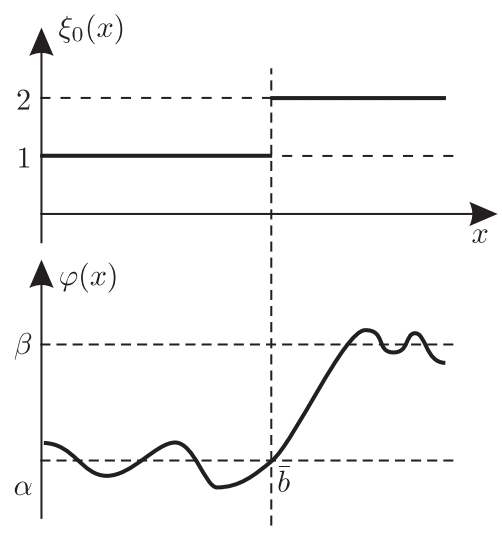

Figure 2.6: Initial data satisfying Condition 2.8

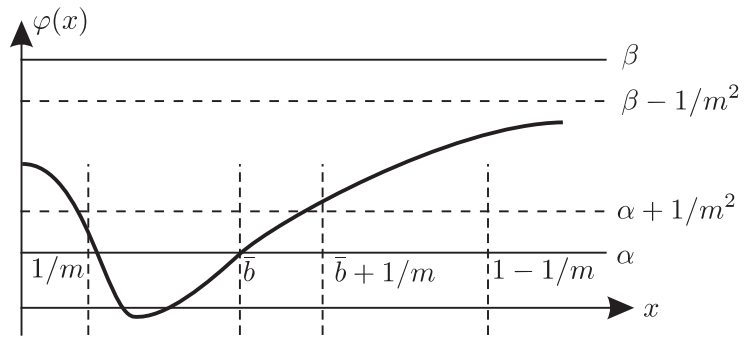

Figure 2.7: Initial data belongs to $E_{m}$

We denote by $E_{m}, m \in \mathbb{N}$, the set of pairs $\left(\varphi, \xi_{0}\right)$ satisfying Condition 2.5 such that $\varphi \in W_{q, N}^{2-2 / q}, \xi_{0}(x)$ is of the form (2.11) and the following hold (see Fig. 2.7):

1. $\bar{b} \in[1 / m, 1-1 / m]$,

2. $\varphi(x) \leq \beta-1 / m^{2}$ for $x \in[0, \bar{b}]$,

3. $\varphi(x) \geq \alpha+1 / m^{2}$ for $x \in[\bar{b}+1 / m, 1]$,

4. if $x \in[\bar{b}, \bar{b}+1 / m]$ and $\varphi(x) \in\left[\alpha, \alpha+1 / m^{2}\right]$, then $\varphi^{\prime}(x) \geq 1 / m$,

5. $\|\varphi\|_{W_{q, N}^{2-2 / q}} \leq m$. 
Note that item 4 in the definition of $E_{m}$ yields

$$
\varphi(x) \geq \alpha+\frac{1}{m}(x-\bar{b}), \quad x \in[\bar{b}, \bar{b}+1 / m] .
$$

This observation easily implies $E_{m} \subset E_{m+1}$. Moreover, Lemma 2.3 below shows that the unity of all sets $E_{m}$ coincides with the set of all transverse data satisfying Condition 2.8. These sets allow us to measure the "level of transversality" of the data. The precise statement is as follows.

Lemma 2.3. 1. Functions $\varphi(x)$ and $\xi_{0}(x)$ satisfy Condition 2.8 if and only if $\left(\varphi, \xi_{0}\right) \in \bigcup_{m=1}^{\infty} E_{m}$.

2. Let $\varphi_{m} \in W_{q, N}^{2-2 / q}$, and let $\xi_{m}(x)$ be defined analogously to (2.11) with some $\bar{b}_{m}$ instead of $\bar{b}$. If

(a) $\left(\varphi_{m}, \xi_{m}\right) \in E_{m} \backslash E_{m-1}, m=2,3, \ldots$,

(b) $\left\|\varphi_{m}-\varphi\right\|_{W_{q, N}^{2-2 / q}} \rightarrow 0$ as $m \rightarrow \infty$ for some $\varphi \in W_{q, N}^{2-2 / q}$,

(c) $\bar{b}_{m}-\bar{b} \rightarrow 0$ as $m \rightarrow \infty$ for some $\bar{b} \in[0,1]$,

then $\bar{b} \in\{0,1\}$ or $\varphi(x)$ is not transverse with respect to $\xi_{0}(x)$, where $\xi_{0}(x)$ is given by (2.11).

3. Let $\left(\varphi, \xi_{0}\right) \in E_{m}$ for some $m \in \mathbb{N}$. Then there is $\varepsilon=\varepsilon(m)>0$ such that $\left(\tilde{\varphi}, \tilde{\xi}_{0}\right) \in E_{m+1}$ whenever $\|\tilde{\varphi}-\varphi\|_{W_{q, N}^{2-2 / q}} \leq \varepsilon,|\tilde{b}-\bar{b}| \leq \varepsilon$, and $\tilde{\xi}_{0}$ is given analogously to (2.11) with $\tilde{b}$ instead of $\bar{b}$.

Proof. 1. Let $\varphi(x)$ and $\xi_{0}(x)$ satisfy Condition 2.8. Items 1,2,5 in the definition of $E_{m}$ directly follow from Condition 2.8 for a sufficiently large $m$.

Further, if $\varphi(\bar{b}) \neq \alpha$, then one can choose $m$ such that $\varphi(x) \notin\left[\alpha, \alpha+1 / m^{2}\right]$ for all points $x \in[\bar{b}, \bar{b}+1 / m]$. In this case item 4 in the definition of $E_{m}$ becomes void and item 3 in the definition of $E_{m}$ is a trivial consequence of item 3 in Condition 2.8

Finally, if $\varphi(\bar{b})=\alpha$, then $\varphi^{\prime}(\bar{b})>0$ and hence there exists $m>0$ such that $\varphi^{\prime}(x) \geq 1 / m$ for $x \in$ $[\bar{b}, \bar{b}+1 / m]$. This inequality implies item 4 of the definition of $E_{m}$ and inequalities (2.12). Applying item 3 of Condition 2.5, we can further increase $m$ so that item 3 hold.

If $\left(\varphi, \xi_{0}\right) \in E_{m}$ for some $m$, then it is obvious that Condition 2.8 holds.

2. Assertion 2 will follow from assertions 1 and 3 . Indeed, if $\bar{b} \in(0,1)$ and $\varphi(x)$ is transverse with respect to $\xi_{0}(x)$, then assertion 1 implies that $\left(\varphi, \xi_{0}\right) \in E_{m_{0}}$ for a sufficiently large $m_{0}$. Therefore, by assertion 3 , $\left(\varphi_{m}, \xi_{m}\right) \in E_{m_{0}+1}$ for all sufficiently large $m$, which contradicts assumption (a) on the sequence $\left(\varphi_{m}, \xi_{m}\right)$.

3. Let us prove assertion 3. Lemma 2.2 implies that

$$
\|\tilde{\varphi}-\varphi\|_{C} \leq c \varepsilon, \quad\left\|\tilde{\varphi}^{\prime}-\varphi^{\prime}\right\|_{C} \leq c \varepsilon, \quad\left\|\tilde{\varphi}^{\prime}\right\|_{C^{\gamma}} \leq c(m+1),
$$

where $c>0$ does not depend on $\varepsilon$ and $m$. Items $1,2,5$ of the definition of $E_{m+1}$ are direct consequence of (2.13), provided that $\varepsilon>0$ is small enough.

Let us prove item 4 in the definition of $E_{m+1}$. Consider $x \in[\tilde{b}, \tilde{b}+1 /(m+1)]$ such that $\tilde{\varphi}(x) \in$ $\left[\alpha, \alpha+1 /(m+1)^{2}\right]$. If $x \geq \bar{b}$, then item 4 is a direct consequence of (2.13). Assume $x<\bar{b}$. Then $|x-\bar{b}| \leq \varepsilon$, and inequalities (2.13) imply

$$
\tilde{\varphi}(\bar{b}) \leq \alpha+\frac{1}{(m+1)^{2}}+|x-\bar{b}| c(m+1) \leq \alpha+\frac{1}{(m+1)^{2}}+\varepsilon c(m+1) .
$$

For small enough $\varepsilon$, this implies that $\varphi(\bar{b}) \leq \alpha+1 / m^{2}$ and hence $\varphi^{\prime}(\bar{b}) \geq 1 / m$. This implies $\tilde{\varphi}^{\prime}(\bar{b}) \geq 1 / m-\varepsilon$ and (by inequalities (2.13)

$$
\tilde{\varphi}^{\prime}(x) \geq \frac{1}{m}-\varepsilon-c(m+1) \varepsilon^{\gamma} .
$$

The latter inequality implies $\tilde{\varphi}^{\prime}(x) \geq 1 /(m+1)$ for small enough $\varepsilon$, which completes the proof of item 4 . 
Let us now prove item 3 in the definition of $E_{m+1}$. Consider $x \in[\tilde{b}+1 /(m+1), 1]$. If $x \geq \bar{b}+1 / m$, then the inequality $\varphi(x) \geq \alpha+1 / m^{2}$ and (2.13) imply $\tilde{\varphi}(x) \geq 1 /(m+1)^{2}$ for small enough $\varepsilon$. Let $x<\bar{b}+1 / m$. The inequality $|\vec{b}-\bar{b}| \leq \varepsilon$ implies $x-\bar{b} \geq 1 /(m+1)-\varepsilon$.

Therefore,

$$
\varphi(x) \geq \alpha+\frac{1}{m}\left(\frac{1}{m+1}-\varepsilon\right) .
$$

Now, using (2.13), we conclude that $\tilde{\varphi}(x) \geq \alpha+1 /(m+1)^{2}$, provided that $\varepsilon>0$ is small enough, which completes the proof of assertion 3.

We will also need the following auxiliary statement.

Lemma 2.4. Let $\left(\varphi, \xi_{0}\right) \in E_{m}$ and $\bar{b} \in(0,1)$ be such that (2.11) holds. Then, for any functions $\psi_{1}, \psi_{2} \in C^{1}$ satisfying

$$
\left\|\psi_{i}-\varphi\right\|_{C}+\left\|\psi_{i}^{\prime}-\varphi^{\prime}\right\|_{C} \leq \frac{1}{4 m^{2}}, \quad i=1,2,
$$

the following holds.

1. The equation $\psi_{i}(x)=\alpha(i=1,2)$ has no more than one root on the interval $[\bar{b}, 1]$.

2. If such a root exists, let us denote it by $a_{i}$; otherwise, we set $a_{i}=\bar{b}$. Then

$$
\begin{aligned}
a_{1}, a_{2} & \in[\bar{b}, \bar{b}+1 / m], \\
\left|a_{1}-a_{2}\right| & \leq 2 m\left\|\psi_{1}-\psi_{2}\right\|_{C} .
\end{aligned}
$$

Proof. Item 1 and inclusions (2.15) are a trivial consequences of items 3 and 4 in the definition of $E_{m}$.

Let us prove item 2. First, let us assume that both $a_{1}, a_{2}$ satisfy $\psi_{1,2}\left(a_{1,2}\right)=\alpha$ (see Fig. 2.8). Without loss of generality, we can assume that $a_{2}>a_{1}$. Let us fix an arbitrarily $x \in\left[a_{1}, a_{2}\right]$. Item 4 in the definition of $E_{m}$ and (2.14) imply that $\psi_{2}(x) \leq 0$ and $\psi_{1}(x) \leq \alpha+1 /\left(2 m^{2}\right)$. Hence, due to (2.14), $\varphi(x) \leq \alpha+1 / m^{2}$, which implies $\varphi^{\prime}(x) \geq 1 / m$ and, thus, $\psi_{1}^{\prime}(x) \geq 1 /(2 m)$. The latter inequality yields

$$
\left|a_{2}-a_{1}\right| \leq 2 m\left|\psi_{1}\left(a_{2}\right)-\psi_{2}\left(a_{2}\right)\right|,
$$

which proves (2.16).

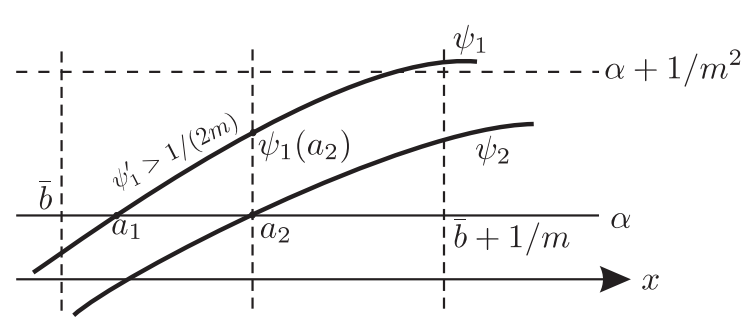

Figure 2.8: Both roots of $\psi_{1,2}(x)=\alpha$ exist

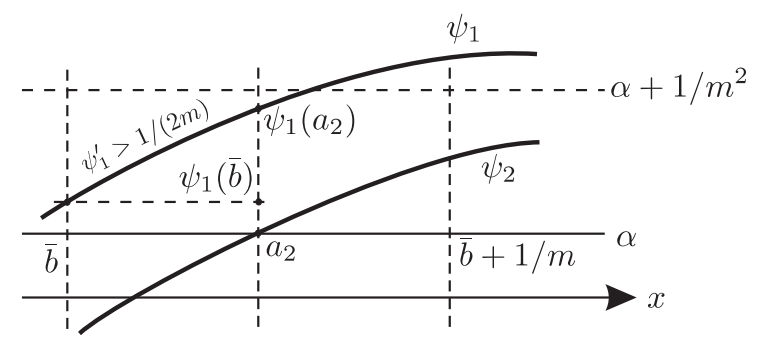

Figure 2.9: A root of $\psi_{2}(x)=\alpha$ exists; $a_{1}=\bar{b}$

Assume that $a_{2}$ satisfies $\psi_{2}\left(a_{2}\right)=\alpha$ and the equation $\psi_{1}(x)=\alpha$ has no roots on the interval $[\bar{b}, 1]$ (see Fig. (2.9). Using inequalities (2.12) and (2.14), we conclude that $\psi_{1}(\bar{b}+1 /(2 m))>0$ and hence $\psi_{1}(\bar{b})>0$. Arguing similarly to the previous case, we conclude that $\psi_{1}^{\prime}(x) \geq 1 /(2 m)$ for $x \in\left[\bar{b}, a_{2}\right]$ and hence

$$
\left|a_{2}-a_{1}\right|=\left|a_{2}-\bar{b}\right| \leq 2 m\left|\psi_{1}\left(a_{2}\right)-\psi_{1}(\bar{b})\right| \leq 2 m\left|\psi_{1}\left(a_{2}\right)-\psi_{2}\left(a_{2}\right)\right|,
$$

which proves (2.16).

If neither of the equations $\psi_{1,2}(x)=\alpha$ has a root on the interval $[\bar{b}, 1]$, then $a_{1}=a_{2}=\bar{b}$ and (2.16) is trivial. 


\section{Auxiliary results}

\subsection{Linear parabolic problem}

In this subsection, we formulate a well-known result on the solvability of a linear parabolic equation in the anisotropic Sobolev space $W_{q}^{2,1}\left(Q_{T}\right)$.

We consider the initial boundary-value problem

$$
\left\{\begin{array}{l}
u_{t}=u_{x x}+F(x, t), \quad(x, t) \in Q_{T}, \\
\left.u_{x}\right|_{x=0}=\left.u_{x}\right|_{x=1}=0, \\
\left.u\right|_{t=0}=\varphi(x), \quad x \in(0,1) .
\end{array}\right.
$$

Combining the results of [10, Chap. 4] (including Lemma 2.1 formulated above in our paper) with Theorem 3.1 in 2 and with the interpolation theory in [14, Secs. 1.14.5, 4.3.3, and 4.4.1], we obtain the following result.

Theorem 3.1. 1. Assume that $q$ and $\gamma$ satisfy (2.1). Fix numbers $T_{0} \geq T>0$. Let $F \in L_{q}\left(Q_{T}\right)$ and $\varphi \in W_{q, N}^{2-2 / q}$. Then problem (3.1) has a unique solution $u \in W_{q}^{2,1}\left(Q_{T}\right)$ and

$$
\begin{gathered}
\|u\|_{W_{q}^{2,1}\left(Q_{T}\right)}+\max _{t \in[0, T]}\|u(\cdot, t)\|_{W_{q, N}^{2-2 / q}} \leq c_{1}\left(\|\varphi\|_{W_{q, N}^{2-2 / q}}+\|F\|_{L_{q}\left(Q_{T}\right)}\right), \\
\|u\|_{C^{\gamma}\left(\bar{Q}_{T}\right)}+\left\|u_{x}\right\|_{C^{\gamma}\left(\bar{Q}_{T}\right)} \leq c_{2}\left(\|\varphi\|_{W_{q, N}^{2-2 / q}}+\|F\|_{L_{q}\left(Q_{T}\right)}\right),
\end{gathered}
$$

The constants $c_{1}, c_{2}>0$ depend on $q, \gamma$, and $T_{0}$, but do not depend on $T, \varphi$, and $F$.

\subsection{Semilinear parabolic problem}

In this subsection, we consider an auxiliary semilinear initial boundary-value problem

$$
\left\{\begin{array}{l}
u_{t}=u_{x x}+f_{0}(u, x, t), \quad(x, t) \in Q_{T}, \\
\left.u_{x}\right|_{x=0}=\left.u_{x}\right|_{x=1}=0 \\
\left.u\right|_{t=0}=\varphi(x), \quad x \in(0,1)
\end{array}\right.
$$

\subsection{1 $\quad E_{\infty, T}$-mild solutions}

First, we assume that $\varphi \in L_{\infty}$. We also assume that the function $f_{0}$ satisfies the following.

1. $f_{0}(u, x, t)$ is measurable with respect to $(x, t) \in[0,1] \times[0, \infty)$ for all $u \in \mathbb{R}$.

2. For every bounded set $B \subset \mathbb{R} \times[0,1] \times[0, \infty)$, there exists a constant $L=L(B)>0$ such that

$$
\begin{gathered}
\left|f_{0}(u, x, t)\right| \leq L \quad \forall(u, x, t) \in B, \\
\left|f_{0}(u, x, t)-f_{0}(v, x, t)\right| \leq L|u-v| \quad \forall(u, x, t),(v, x, t) \in B .
\end{gathered}
$$

We give one result from [12] on the so-called $E_{\infty, T}$-mild solutions of problem (3.2).

First, we define the operator $A_{0}: D\left(A_{0}\right) \subset L_{p} \rightarrow L_{p}$ for $p>1$ by

$$
\begin{gathered}
D\left(A_{0}\right)=\left\{\psi \in C^{2}: \psi^{\prime}(0)=\psi^{\prime}(1)=0\right\}, \\
A_{0} \psi=-\psi_{x x}+\psi \quad \forall \psi \in D\left(A_{0}\right) .
\end{gathered}
$$

The operator $A_{0}$ has a closure $A_{p}$ in $L_{p}$. The operators $A_{p}$ are generators of analytic semigroups $S_{p}(t)$ in $L_{p}$. By Lemma 1 in [12, p. 15], $S_{p_{1}}(t) \subset S_{p_{2}}(t)$ for any $p_{1}, p_{2} \in(1, \infty), p_{1} \geq p_{2}$. By Lemma 2 in [12, p. 19],

$$
\sup _{t \in[0, T]}\left\|S_{p}(t) \psi\right\|_{L_{\infty}} \leq\|\psi\|_{L_{\infty}} \quad \forall \psi \in L_{\infty} .
$$


Therefore, one can define the operators $P(t)$ as the restrictions of $S_{p}(t)$ to the space $L_{\infty}$. These operators do not depend on $p$ and are continuous from $L_{\infty}$ to $L_{\infty}$. Furthermore, since

$$
P\left(t_{1}+t_{2}\right)=P\left(t_{1}\right) P\left(t_{2}\right) \quad \forall t_{1}, t_{2} \in[0, \infty)
$$

they define a semigroup in $L_{\infty}$.

Note that it is not a strongly continuous semigroup in $L_{\infty}$ because, due to Lemma 2 in [12, p. 19], $P(t) \psi \rightarrow \psi$ in $L_{\infty}$ as $t \rightarrow 0$ if and only if $\psi \in C$.

Definition 3.1. Let $T \in(0, \infty]$. A $E_{\infty, T}$-mild solution of problem (3.2) for initial data $\varphi \in L_{\infty}$ on the time interval $[0, T)$ is a measurable function $u(x, t),(x, t) \in(0,1) \times(0, T)$, satisfying

$$
\begin{gathered}
u(\cdot, t) \in L_{\infty}, \quad \sup _{s \in(0, t)}\|u(\cdot, s)\|_{L_{\infty}}<\infty \quad \forall t \in(0, T), \\
u(\cdot, t)=P(t) \varphi+\int_{0}^{t} P(t-s)\left(f_{0}(u(\cdot, s), \cdot, s)+u(\cdot, s)\right) d s \quad \forall t \in(0, T),
\end{gathered}
$$

where the integral is an absolutely converging Bochner inegral in $L_{\infty}$.

Definition 3.2. We say that $T \in(0, \infty)$ is a maximal existence time for a given initial data $\varphi$ if problem (3.2) has a $E_{\infty, T}$-mild solution on the interval $[0, T)$, but for any $T^{\prime}>T$, it has no $E_{\infty, T^{\prime}}$-mild solution on the interval $\left[0, T^{\prime}\right)$.

The following lemma is formulated as Theorem 1 in [12, p. 111].

Lemma 3.1. Assume that $\varphi \in L_{\infty}$ and the above conditions on $f_{0}$ are satisfied. Then there exists a maximal existence time $T \in(0, \infty]$ and problem (3.2) has a unique $E_{\infty, T}$-mild solution on the interval $[0, T)$.

If the maximal existence time $T$ is finite, then

$$
\lim _{t \rightarrow T}\|u(\cdot, t)\|_{L_{\infty}}=\infty
$$

\subsubsection{Uniformly bounded solutions}

Now we formulate a result which states that if problem (3.2) has a solution $u \in W_{q}^{2,1}\left(Q_{T}\right)$, then it is bounded uniformly with respect to $T \in(0, \infty)$. It can be proved by regularizing the right-hand side and applying the invariant-rectangles method, which can also be exploit for systems of reaction-diffusion equations (see, e.g., [13]).

Lemma 3.2. Let $\varphi \in W_{q, N}^{2-2 / q}, u \in W_{q}^{2,1}\left(Q_{T}\right)$, and $F(x, t)=f_{0}(u(x, t), x, t)$ belongs to $L_{q}\left(Q_{T}\right)$. We assume that $u$ satisfies (3.2) and the following hold for some $U>0$.

1. $f_{0}(\cdot, x, t)$ is continuous at the points $\pm U$ uniformly with respect to $(x, t) \in \bar{Q}_{T}$ (perhaps, after modification on a subset of $\bar{Q}_{T}$ of measure zero),

2. $f_{0}(U, x, t)<0, f_{0}(-U, x, t)>0$ for a.e. $(x, t) \in \bar{Q}_{T}$,

3. $\|\varphi\|_{C}<U$.

Then $\|u\|_{C\left(\bar{Q}_{T}\right)}<U$. 


\subsection{Semilinear parabolic problem with a special nonlinearity}

In this subsection, we specify the nonlinearity $f_{0}(u, x, t)$ in (3.2).

Let us fix initial data $\varphi$ and $\xi_{0}$ satisfying Condition 2.8. By Lemma $2.3,\left(\varphi, \xi_{0}\right) \in E_{m}$ for a sufficiently large $m$. Let us fix such an $m$ (see Fig 2.7). Next, we choose a number $U>0$ such that Condition 2.4 holds for $U$ and

$$
\|\varphi\|_{C}<U
$$

Further, we fix a number $T>0$ and consider functions $u_{0} \in C\left(\bar{Q}_{T}\right)$ and $b_{0} \in C[0, T]$ with the following properties:

$$
\begin{gathered}
\left\|u_{0}\right\|_{C\left(\bar{Q}_{T}\right)} \leq U, \\
b_{0}(t) \in[\bar{b}, \bar{b}+1 / m], \quad t \in[0, T] .
\end{gathered}
$$

Now we define the function $f_{0}(u, x, t)$ by

$$
\begin{gathered}
f_{0}(u, x, t)=f\left(u, v_{0}(x, t)\right), \\
v_{0}(x, t)= \begin{cases}H_{1}\left(u_{0}(x, t)\right), & 0 \leq x \leq b_{0}(t), \\
H_{2}\left(u_{0}(x, t)\right), & b_{0}(t)<x \leq 1,\end{cases}
\end{gathered}
$$

where we use convention (2.2).

Note that, in general, $v_{0}(x, t) \neq \mathcal{H}\left(\xi_{0}(x), u_{0}(x, \cdot)\right)(t)$. However, the following is true and will be essentially used later on.

Remark 3.1. Suppose that the following hold for each $t \in[0, T]$.

1. $u_{0}(x, 0)=\varphi(x)$.

2. The equation $u(x, t)=\alpha$, on the interval $[\bar{b}, 1]$ has no more than one root. Let us define a function $a_{0}(t)$ as follows: if the above root exists, then $a_{0}(t)$ is equal to this root, and $a_{0}(t)=\bar{b}$ otherwise.

3. The function

$$
b_{0}(t)=\max _{s \in[0, t]} a_{0}(s)
$$

satisfies $b_{0}(t) \in[\bar{b}, \bar{b}+1 / m]$.

4. The equation $u_{0}(x, t)=\beta$ on the interval $\left[0, b_{0}(t)\right]$ has no roots.

Then the nonlinearity $v_{0}(x, t)$ given by (3.7) coincides with the spatially distributed hysteresis defined for $u_{0}(x, t)$ :

$$
v_{0}(x, t) \equiv \mathcal{H}\left(\xi_{0}(x), u_{0}(x, \cdot)\right)(t) .
$$

The next lemma establishes basic properties of the "max"-operator in (3.8).

Lemma 3.3. Let $\lambda=0$ or $\gamma$. Then the following hold.

1. If $a \in C^{\lambda}[0, T]$ and $b(t)=\max _{s \in[0, t]} a(s)$, then $b \in C^{\lambda}[0, T]$ and

$$
\|b\|_{C^{\lambda}[0, T]} \leq\|a\|_{C^{\lambda}[0, T]} .
$$

2. If $a_{j} \in C[0, T]$ and $b_{j}(t)=\max _{s \in[0, t]} a_{j}(s), j=1,2$, then

$$
\left\|b_{1}-b_{2}\right\|_{C[0, T]} \leq\left\|a_{1}-a_{2}\right\|_{C[0, T]} .
$$

Proof. We leave details to the reader. 
In what follows, we will need the continuous dependence of $v_{0}$ defined by (3.7) on $u_{0}$ and $b_{0}$. Denote by $\mathcal{R}$ the set of pairs $\left(u_{0}, b_{0}\right) \in C\left(\bar{Q}_{T}\right) \times C[0, T]$ satisfying conditions (3.4) and (3.5).

Lemma 3.4. For any $\left(u_{0}, b_{0}\right),\left(\hat{u}_{0}, \hat{b}_{0}\right) \in \mathcal{R}(m \in \mathbb{N})$, let $v_{0}$ be defined by (3.7) and $\hat{v}_{0}$ by (3.7), where $u_{0}$ and $b_{0}$ are replaced by $\hat{u}_{0}$ and $\hat{b}_{0}$, respectively. Then, for any $p \in[1, \infty)$,

$$
\left\|v_{0}-\hat{v}_{0}\right\|_{L_{p}\left(Q_{T}\right)} \leq c_{0}\left(T^{1 / p}\left\|u_{0}-\hat{u}_{0}\right\|_{C\left(\bar{Q}_{T}\right)}^{\sigma}+\left\|b_{0}-\hat{b}_{0}\right\|_{L_{1}(0, T)}^{1 / p}\right),
$$

where $\sigma$ is the constant in Condition 2.1 and $c_{0}>0$ depends on $U$ and $p$, but does not depend on $u_{0}, b_{0}, T$.

Proof. We fix some $t \in[0, T]$ and assume that $b_{0}(t) \leq \hat{b}_{0}(t)$ for this $t$. Then, using (3.7) and omitting the arguments of the integrands, we have

$$
\begin{aligned}
\int_{0}^{1}\left|v_{0}-\hat{v}_{0}\right|^{p} d x & =\int_{0}^{b_{0}(t)}\left|H_{1}\left(u_{0}\right)-H_{1}\left(\hat{u}_{0}\right)\right|^{p} d x+\int_{\hat{b}_{0}(t)}^{1}\left|H_{2}\left(u_{0}\right)-H_{2}\left(\hat{u}_{0}\right)\right|^{p} d x \\
& +\int_{b_{0}(t)}^{\hat{b}_{0}(t)}\left|H_{2}\left(u_{0}\right)-H_{1}\left(\hat{u}_{0}\right)\right|^{p} d x .
\end{aligned}
$$

Using Condition 2.1] in the first two integrals and the boundedness of $H_{j}(u)$ for $|u| \leq U$ in the second integral, we obtain

$$
\int_{0}^{1}\left|v_{0}-\hat{v}_{0}\right|^{p} d x \leq k_{1}\left(\left\|u_{0}-\hat{u}_{0}\right\|_{C\left(\bar{Q}_{T}\right)}^{\sigma p}+k_{2}\left|b_{0}(t)-\hat{b}_{0}(t)\right|\right),
$$

where $k_{1}>0$ depends on $U$ and $p$, but does not depend on $u_{0}, b_{0}, T$.

Integrating the latter inequality with respect to $t$ from 0 to $T$ yields (3.9).

Denote

$$
V=\max _{|u| \leq U}\left\{H_{1}(u), H_{2}(u)\right\}, \quad f_{U}=\max _{|u| \leq U,|v| \leq V}|f(u, v)| .
$$

The main step in finding a solution of problem (2.6)-(2.8) is the following theorem.

Theorem 3.2. Let $f_{0}$ be defined by (3.6), and let $q$ and $\gamma$ satisfy (2.1). We fix an arbitrary $T_{0}>0$. Then the following hold.

1. Problem (3.2) has a unique solution $u \in W_{q}^{2,1}\left(Q_{T}\right)$ and, for any $T \leq T_{0}$,

$$
\begin{gathered}
\|u\|_{C\left(\bar{Q}_{T}\right)}<U, \\
\|u\|_{W_{q}^{2,1}\left(Q_{T}\right)}+\max _{t \in[0, T]}\|u(\cdot, t)\|_{W_{q, N}^{2-2 / q}} \leq c_{1}\left(\|\varphi\|_{W_{q, N}^{2-2 / q}}+f_{U}\right), \\
\|u\|_{C^{\gamma}\left(\bar{Q}_{T}\right)}+\left\|u_{x}\right\|_{C^{\gamma}\left(\bar{Q}_{T}\right)} \leq c_{2}\left(\|\varphi\|_{W_{q, N}^{2-2 / q}}+f_{U}\right),
\end{gathered}
$$

where $f_{U}$ is given by (3.10) and $c_{1}, c_{2}>0$ depend only on $T_{0}$ and do not depend on $m, u_{0}, b_{0}, \varphi, u, T$.

2. The solution of problem (3.2) continuously depends on $\varphi, u_{0}$ and $b_{0}$. In other words, if $u_{n} \in W_{q}^{2,1}\left(Q_{T}\right)$, $n=1,2, \ldots$, are solutions of problem (3.2) with $\varphi, u_{0}, b_{0}, v_{0}$ replaced by $\varphi_{n}, u_{0 n}, b_{0 n}, v_{0 n}$, then

$$
\left\|u_{n}-u\right\|_{W_{q}^{2,1}\left(Q_{T}\right)} \rightarrow 0, \quad n \rightarrow \infty
$$

whenever

$$
\left\|\varphi_{n}-\varphi\right\|_{W_{q, N}^{2-2 / q}}+\left\|u_{0 n}-u_{0}\right\|_{C\left(\bar{Q}_{T}\right)}+\left\|b_{0 n}-b_{0}\right\|_{C[0, T]} \rightarrow 0, \quad n \rightarrow \infty .
$$


3. There is a number $T=T(m) \in\left(0, T_{0}\right]$ and a natural number $N=N(m, U) \geq m$ which do not depend on $u_{0}, b_{0}, \varphi, u$, such that, for any $t \in[0, T]$, the following is true.

(a) The equation $u(x, t)=\alpha$ on the interval $[\bar{b}, 1]$ has no more than one root. If this root exists, we denote it by $a(t)$; otherwise, we set $a(t)=\bar{b}$ (similarly to Lemma 2.4). In this case, $a(t) \in$ $[\bar{b}, \bar{b}+1 / N], a \in C^{\gamma}[0, T]$, and

$$
\|a\|_{C^{\gamma}[0, T]} \leq a^{*},
$$

where $a^{*}>0$ depends on $m$, but does not depend on $u_{0}, b_{0}, \varphi$.

(b) The hysteresis $\mathcal{H}\left(\xi_{0}, u\right)$ and its configuration function $\xi(x, t)$ have exactly one discontinuity point $b(t) ;$ moreover, $b(t)=\max _{s \in[0, t]} a(s)$ and $b \in C^{\gamma}[0, T]$.

(c) $(u(\cdot, t), \xi(\cdot, t)) \in E_{N}$.

Proof. 1. Throughout the proof, we assume that $u_{0}$ and $b_{0}$ are extended as continuous functions to $\left[0, T_{0}\right]$ in such a way that (3.4) and (3.5) hold on this interval.

It follows from the definition (3.6) of the function $f_{0}$ and from the Lipschitz continuity of the function $f$ (Condition 2.3) that the function $f_{0}(u, x, t)$ satisfies assumptions 1 and 2 in Sec. 3.2 . Therefore, by Lemma 3.1. there is $T_{1} \in\left(0, T_{0}\right]$ such that problem (3.2) has a unique $E_{\infty, T_{1}}$-mild solution. Hence, $f_{0}(u(x, t), x, t)$ is in $L_{\infty}\left(Q_{T_{1}}\right)$, and Theorem 3.1 yields $u \in W_{q}^{2,1}\left(Q_{T_{1}}\right)$.

Now we claim that

$$
\|u\|_{C\left(\bar{Q}_{T_{1}}\right)}<U .
$$

Indeed, the function $v_{0}(x, t)$ in (3.6) is bounded for $(x, t) \in \bar{Q}_{T_{1}}$; therefore, $f_{0}(u, x, t)=f\left(u, v_{0}(x, t)\right)$ satisfies assumption 1 in Lemma 3.2 due to the uniform continuity of $f(u, v)$ on bounded sets. Assumption 2 in Lemma 3.2 holds due to Condition 2.4 and the choice of $u_{0}$ in (3.4). Assumption 3 in Lemma 3.2 holds because of the choice of $U$ (see (3.3)). Thus, Lemma 3.2 implies estimate (3.16).

Combining Lemma 3.1 with estimate (3.16), we see that $T_{1}$ can be chosen equal to $T_{0}$. Hence, $u \in$ $W_{q}^{2,1}\left(Q_{T_{0}}\right)$ and, by Theorem 3.1 and (3.16) (with any $T \leq T_{0}$ instead of $T_{1}$ ), we obtain estimates (3.12). Part 1 of the theorem is proved.

2. Let us prove (3.13). Assume the contrary: there is $\varepsilon>0$ and a subsequence of $u_{n}$ (which we denote $u_{n}$ again) such that

$$
\left\|u_{n}-u\right\|_{W_{q}^{2,1}\left(Q_{T}\right)} \geq \varepsilon, \quad n=1,2, \ldots
$$

2a. Note that (3.14) and Lemma 3.4 imply

$$
\left\|v_{0 n}-v_{0}\right\|_{L_{q}\left(Q_{T}\right)} \rightarrow 0, \quad n \rightarrow \infty
$$

Further, by part 1 of the theorem, $u_{n}$ are bounded in $W_{q}^{2,1}\left(Q_{T}\right)$ uniformly with respect to $n$. Therefore, by the compactness of the embedding $W_{q}^{2,1}\left(Q_{T}\right) \subset L_{q}\left(Q_{T}\right)$, there is a subsequence of $u_{n}$ (which we denote $u_{n}$ again) that is fundamental in $L_{q}\left(Q_{T}\right)$.

For this subsequence, using Theorem 3.1 and the Lipschitz continuity of $f$ (Condition 2.3), we have

$$
\begin{aligned}
& \left\|u_{n}-u_{k}\right\|_{W_{q}^{2,1}\left(Q_{T}\right)} \\
& \leq k_{1}\left(\left\|\varphi_{n}-\varphi_{k}\right\|_{W_{q, N}^{2-2 / q}}+\left(\int_{Q_{T}}\left|f\left(u_{n}, v_{0 n}\right)-f\left(u_{k}, v_{0 k}\right)\right|^{q} d x d t\right)^{1 / q}\right) \\
& \leq k_{2}\left(\left\|\varphi_{n}-\varphi_{k}\right\|_{W_{q, N}^{2-2 / q}}+\left(\int_{Q_{T}}\left(\left|u_{n}-u_{k}\right|+\left|v_{0 n}-v_{0 k}\right|\right)^{q} d x d t\right)^{1 / q}\right) \\
& \leq k_{2}\left(\left\|\varphi_{n}-\varphi_{k}\right\|_{W_{q, N}^{2-2 / q}}+\left\|u_{n}-u_{k}\right\|_{L_{q}\left(Q_{T}\right)}+\left\|v_{0 n}-v_{0 k}\right\|_{L_{q}\left(Q_{T}\right)}\right),
\end{aligned}
$$


where $k_{1}, k_{2}>0$ do not depend on $n$ and $k$. The latter inequality, the first convergence in (3.14), relation (3.18), and the fact that $u_{n}$ is fundamental in $L_{q}\left(Q_{T}\right)$ imply that $u_{n}$ is fundamental and, thus, converges to some $\hat{u}$ in $W_{q}^{2,1}\left(Q_{T}\right)$.

2b. Passing to the limit as $n \rightarrow \infty$ and using Lemma 3.4 and Condition 2.3. we conclude that $\hat{u}$ is a solution of the problem

$$
\left\{\begin{array}{l}
\hat{u}_{t}=\hat{u}_{x x}+f\left(\hat{u}, v_{0}\right), \quad(x, t) \in Q_{T}, \\
\left.\hat{u}_{x}\right|_{x=0}=\left.\hat{u}_{x}\right|_{x=1}=0, \\
\left.\hat{u}\right|_{t=0}=\varphi(x), \quad x \in(0,1) .
\end{array}\right.
$$

But the latter problem has a unique solution due to part 1 of the proof. Hence, $\hat{u}=u$, which contradicts (3.17).

Part 2 is proved.

3. Denote

$$
\Omega=\left\{(x, \varphi(x)): x \in[\bar{b}, \bar{b}+1 / m], \varphi(x) \in\left[\alpha, \alpha+1 / m^{2}\right]\right\} .
$$

Consider the two cases: $\Omega \neq \varnothing$ and $\Omega=\varnothing$.

Case I. Let $\Omega \neq \varnothing$. The second inequality in (3.12) implies that

$$
\left\|u\left(\cdot, t_{1}\right)-u\left(\cdot, t_{2}\right)\right\|_{C}+\left\|u_{x}\left(\cdot, t_{1}\right)-u_{x}\left(\cdot, t_{2}\right)\right\|_{C} \leq c\left|t_{1}-t_{2}\right|^{\gamma}
$$

where $c=c_{2}\left(m+f_{U}\right)$. This inequality implies that, for small enough $\tau(m)>0$, the functions $\varphi, \psi_{1}=u\left(\cdot, t_{1}\right)$, $\psi_{2}=u\left(\cdot, t_{2}\right)$ with $t_{1}, t_{2} \in[0, \tau(m)]$ satisfy the assumptions of Lemma 2.4 Therefore, $a \in C^{\gamma}$ and

$$
\|a\|_{C^{\gamma}} \leq a^{*}=1+2 m c .
$$

Denote $b(t)=\max _{s \in[0, t]} a(s)$. Note that $b(0)=a(0)=\bar{b}$. It follows from (3.21) and (3.22) (decreasing $\tau(m)$ if necessarily) that

$$
\begin{gathered}
b(t) \in[\bar{b}, \bar{b}+1 /(2 m)], \quad\|b\|_{C^{\gamma}[0, T]} \leq a^{*}, \\
u_{x}(x, t) \geq \frac{1}{m+1} \quad \forall x \in[b(t), b(t)+1 /(2 m)] .
\end{gathered}
$$

Denote $N=N(m, U)=\max \left(2 m,\left[c_{1}\left(m+f_{U}\right)\right]+1\right)$, where $c_{1}$ is the constant in (3.12) and [.] stands for the integer part of a number. Then (3.23) and (3.24) imply

$$
\begin{gathered}
a(t), b(t) \in[\bar{b}, \bar{b}+1 / N], \\
u_{x}(x, t) \geq \frac{1}{N} \quad \forall x \in[b(t), b(t)+1 / N] .
\end{gathered}
$$

Now we introduce the function $\xi(x, t)$ as follows: $\xi(x, t)=1$ for $x \leq b(t)$ and $\xi(x, t)=2$ for $x>b(t)$. We will see below that $\xi(x, t)$ is the configuration function of the hysteresis $H\left(\xi_{0}, u\right)$.

Let us show that $(u(\cdot, t), \xi(\cdot, t)) \in E_{N}$ on the interval $t \in[0, T]$, provided that $T=T(m)$ is small enough.

i. $b(t) \leq 1-1 / N$. This follows from (3.23).

ii. $u(x, t) \leq \beta-1 / N^{2}$ for $x \in[0, \bar{b}]$. This follows for sufficiently small $T$ from the fact that $u(x, 0)=$ $\varphi(x) \leq \beta-1 / m^{2}$ for $x \in[0, \bar{b}]$ and from the Hölder continuity of $u$ (the second inequality in (3.12) $)$.

iii. $u(x, t) \geq \alpha+1 / N^{2}$ for $x \in[\bar{b}+1 / N, 1]$. This follows for sufficiently small $T$ from the fact that $u(x, 0)=\varphi(x) \geq \alpha+1 /(N m)$ for $x \in[\bar{b}+1 / N, 1]$ and from the Hölder continuity of $u$ (the second inequality in $(3.12)$ ).

iv. If $x \in[b(t), b(t)+1 / N]$, then $u(x, t) \geq \alpha$ and $u_{x}(x, t) \geq 1 / N$. The first inequality holds by construction of the function $b(t)$. The second inequality follows from (3.24). 
v. $\|u(\cdot, t)\|_{W_{q, N}^{2-2 / q}} \leq N$. This follows for sufficiently small $T$ from the fact that $\|\varphi\|_{W_{q, N}^{2-2 / q}} \leq m$ and from the first inequality in (3.12).

Items $\mathrm{i}-\mathrm{v}$ together with relations (3.22), (3.23), and (3.25) prove assertions 3.(a)-3.(c) of the theorem in Case I.

Case II. Let $\Omega=\varnothing$, where $\Omega$ is the set in (3.20). Since $u(x, 0)=\varphi(x) \geq 1 / m^{2}$ for $x \in[\bar{b}, \bar{b}+1 / m]$, it follows from the Hölder continuity of $u$ (the second inequality in (3.12)) that $u(x, t) \geq 1 /(m+1)^{2}$ for $x \in[\bar{b}, \bar{b}+1 /(m+1)]$, provided that $T$ is sufficiently small.

In this case $a(t)=\bar{b}$ for all $t \in[0, T]$ and it is easy to see that parts 1-5 of the definition of $E_{N}$ again hold for the pair $(u(\cdot, t), \xi(\cdot, t))$ and for $N=m+1$ on the interval $t \in[0, T]$, provided that $T$ is small enough.

Thus, we have proved assertions 3.(a)-3.(c) of the theorem in Case II.

Remark 3.2. Let

$$
f_{0}(u, x, t)=f\left(u, v_{0}(x, t)\right), \quad(x, t) \in Q_{T_{0}},
$$

where $v_{0}(x, t)$ is the spatially distributed hysteresis defined for some $u_{0} \in C\left(\bar{Q}_{T}\right)$ :

$$
v_{0}(x, t)=\mathcal{H}\left(\xi_{0}(x), u_{0}(x, \cdot)\right)(t) .
$$

Let

$$
\left\|u_{0}\right\|_{C\left(\bar{Q}_{T_{0}}\right)} \leq U
$$

and the function $v_{0}(x, t)$ be measurable. Then parts 1 and 3 of Theorem 3.2 remain true. The proof is analogous to that for Theorem 3.2

\section{Proof of the main results}

\subsection{Existence of solutions}

In this subsection, we prove an analogue of Theorem 2.1 under the assumptions made in Sec. 2.5.

As before, we choose $m$ such that $\left(\varphi, \xi_{0}\right) \in E_{m}$. It will be convenient to reformulate the theorem.

Theorem 4.1 (local existence). Let Condition 2.8 be satisfied. Then the following hold for the number $T=T(m)>0$ from part 3 of Theorem 3.2 .

1. Any solution $u \in W_{q}^{2,1}\left(Q_{T}\right)$ of problem (2.6)-(2.8) in $Q_{T}$ is transverse and preserves spatial topology. Moreover, it possesses all the properties from part 3 of Theorem 3.2

2. There is at least one transverse topology preserving solution $u \in W_{q}^{2,1}\left(Q_{T}\right)$ of problem (2.6)-(2.8) in $Q_{T}$.

Proof. 1. Let $u \in W_{q}^{2,1}\left(\bar{Q}_{T}\right)$ be an arbitrary solution of problem (2.6)-(2.8). Using Lemma 3.2 (as in the proof of Theorem 3.2), we obtain that $\|u\|_{C\left(\bar{Q}_{T}\right)}<U$. Therefore, by Remark 3.2, the first assertion of the theorem is true.

2. Let us prove the second assertion. Let $\mathcal{R} \subset C\left(\bar{Q}_{T}\right) \times C[0, T]$ be the set defined before Lemma 3.4 Clearly, $\mathcal{R}$ is a closed convex set.

Take any $\left(u_{0}, b_{0}\right) \in \mathcal{R}$ and define $f_{0}(u, x, t)$ by formula (3.6). Then Theorem 3.2 implies that problem (3.2) has a unique transverse topology preserving solution $u \in W_{q}^{2,1}\left(Q_{T}\right) \subset C^{\gamma}\left(\bar{Q}_{T}\right)$.

Consider the functions $a(t)$ and $b(t)$ (see part 3 of Theorem [3.2). By Theorem [3.2, $a, b \in C^{\gamma}[0, T]$ and the function $b(t)$ defines the (unique) discontinuity point of the hysteresis $\mathcal{H}\left(\xi_{0}, u\right)$ and of its configuration function $\xi(x, t)$ at each moment $t \in[0, T]$. Moreover $(u, b) \in \mathcal{R}$.

Thus, we can define a nonlinear operator $R: \mathcal{R} \rightarrow \mathcal{R}$ by the formula $R\left(u_{0}, b_{0}\right)=(u, b)$. 
Let us show that $R$ is continuous. Let a sequence $\left(u_{0 n}, b_{0 n}\right)$ converge to $\left(u_{0}, b_{0}\right)$. We define $f_{0 n}(u, x, t)$ by formula (3.6) with $u_{0 n}, b_{0 n}, v_{0 n}$ instead of $u_{0}, b_{0}, v_{0}$. Let $u_{n}$ be a solution of problem (3.2) with the right-hand side $f_{0 n}$. By Theorem 3.2, there is a number $N=N(m, U) \in \mathbb{N}$ such that

$$
(u, \xi),\left(u_{n}(\cdot, t), \xi_{n}(\cdot, t)\right) \in E_{N} \quad \forall t \in[0, T], n=1,2, \ldots
$$

Let $a_{n}(t)$ corresponds to $u_{n}(x, t)$ in the same way as $a(t)$ corresponds to $u(x, t)$. Set $b_{n}(t)=\max _{s \in[0, t]} a_{n}(s)$ for $t \in[0, T]$. Then

$$
R\left(u_{0 n}, b_{0 n}\right)=\left(u_{n}, b_{n}\right) .
$$

By the construction of $T(\leq \tau(m))$ in the proof of assertion 3 of Theorem 3.2 the functions $\varphi, \psi_{1}=u(\cdot, t)$, $\psi_{2}=u_{n}(\cdot, t)$ satisfy the assumptions of Lemma 2.4 and, hence,

$$
\left|a_{n}(t)-a(t)\right| \leq 2 m\left\|u_{n}-u\right\|_{C\left(\bar{Q}_{T}\right)} .
$$

This implies that $a_{n}$ converges to $a$ in $C[0, T]$. Thus, Lemma 3.3 implies that $b_{n}$ converges to $b$ in $C[0, T]$. Therefore, the operator $R$ is continuous on $\mathcal{R}$.

The operator $R$ is also compact. Indeed, the map $\mathcal{R} \ni\left(u_{0}, b_{0}\right) \mapsto u \in C\left(\bar{Q}_{T}\right)$ is compact due to (3.12) and the compactness of the embedding $C^{\gamma}\left(\bar{Q}_{T}\right) \subset C\left(\bar{Q}_{T}\right)$. The map $\mathcal{R} \ni\left(u_{0}, b_{0}\right) \mapsto b \in C[0, T]$ is compact due to (3.15), part 1 of Lemma 3.3, and the compactness of the embedding $C^{\gamma}[0, T] \subset C[0, T]$.

Now the Schauder fixed-point theorem implies that the operator $R$ has a fixed point $(u, b) \in \mathcal{R}$. Taking into account Remark 3.1 and (4.1), we see that $u$ is a transverse topology preserving solution of problem (2.6)(2.8).

\subsection{Continuation of solutions}

In this subsection, we prove an analogue of Theorem 2.2 under the assumptions made in Sec. 2.5.

Proof of Theorem 2.2. 1. We assume that there is $T_{0}>0$ such that $u(x, t)$ cannot be continued to $\left[0, T_{0}\right]$ as a transverse solution of problem (2.6) $-(2.8)$.

Applying Theorem 4.1 and using part 1 of Lemma (2.3), we obtain a sequence $m_{k} \in \mathbb{N}(k=1,2, \ldots)$ such that $m_{k+1}>m_{k}$ and a sequence of time moments $t_{k}(k=1,2, \ldots)$ such that $t_{k+1}>t_{k}$ with the following properties.

1. For each $k$, the solution $u(x, t)$ of problem (2.6)-(2.8) can be continued as a transverse solution to the time interval $\left[0, t_{k}\right]$.

2. For each $k$,

$$
u\left(\cdot, t_{k}\right) \in E_{m_{k}} \backslash E_{m_{k}-1} .
$$

Denote $T=\lim _{k \rightarrow \infty} t_{k}$. By assumption, $T \leq T_{0}$.

Since $u$ is a solution of problem (2.6) (2.8) in $Q_{t_{k}}$ for all $k$ and

$$
\|u\|_{W_{q}^{2,1}\left(Q_{t_{k}}\right)} \leq c_{1}\left(\|\varphi\|_{W_{q, N}^{2-2 / q}}+f_{U}\right)
$$

by Remark 3.2 (with the right-hand side not depending on $k$ ), it follows that $u \in W_{q}^{2,1}\left(Q_{T}\right)$ and $u$ is a solution of problem (2.6) -(2.8) in $Q_{T}$. Since $u(\cdot, t)$ is a continuous $W_{q, N}^{2-2 / q}$-valued function, we have

$$
\left\|u\left(, t_{k}\right)-u(\cdot, T)\right\|_{W_{q, N}^{2-2 / q}} \rightarrow 0, \quad k \rightarrow \infty .
$$

Denote by $b(t)$ the discontinuity point of the configuration function $\xi(x, t)$. By construction $b(t)$ is continuous and nondecreasing on $\left[0, t_{k}\right]$ for all $k$. Therefore, $b(t)$ is continuous on $[0, T]$. In particular,

$$
b\left(t_{k}\right)-b(T) \rightarrow 0, \quad k \rightarrow \infty .
$$


It follows from (4.2)-(4.4) and from part 2 of Lemma 2.3 that $b(T)=1$ or $u(x, T)$ is not transverse with respect to $\xi(x, T)$.

2. Now we consider several cases.

2.1. First, we assume that $b(T)<1$. Then $u(x, T)$ is not transverse with respect to $\xi(x, T)$. This happens because the graph of the function $u(x, T)$ touches the line $\alpha$ or $\beta$ (see Fig. 4.1), or because $u(b(T), T)=\alpha$, $u_{x}(b(T), T)=0$ (see Fig. 4.2).
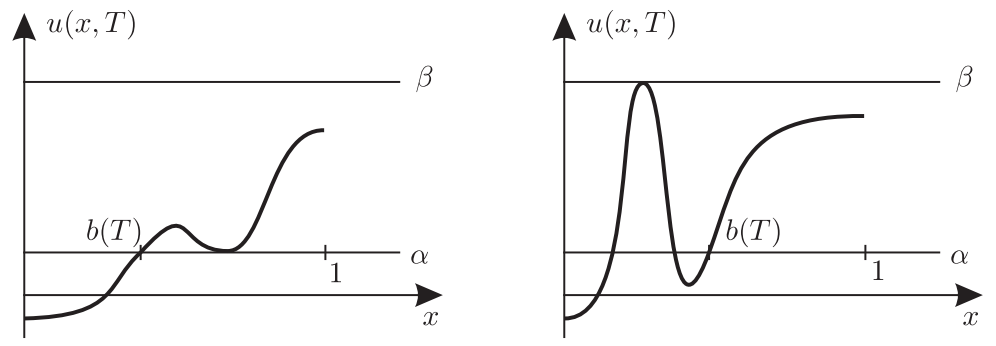

Figure 4.1: Transversality fails at a point different from $b(T)$

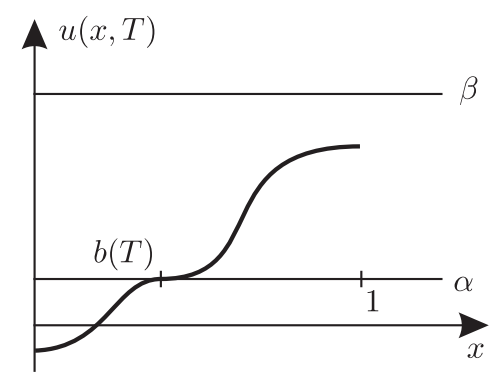

Figure 4.2: Transversality fails at the point $b(T) \neq 1$

2.2. If $b(T)=1$, then $u(1, T)=\alpha$. Furthermore, $u_{x}(1, T)=0$ due to the Neumann boundary condition (2.7). In this case, either $u(x, T)$ is not transverse (if the transversality fails at some point $x \in[0,1)$ at the same moment $t=T$, in which case $\left.T=T_{\max }\right)$ or $u(x, T)$ is transverse and $\xi(x, T) \equiv 2, x \in[0,1]$. In the latter case, we can proceed similarly to the above, but effectively without hysteresis, i.e.,

$$
\mathcal{H}\left(\xi_{0}(x), u(x, \cdot)\right)(t) \equiv H_{2}(u(x, t)), \quad t \geq T,
$$

(see Fig. 4.3). Thus, one can find $T_{\max }$ as in part 1 of the proof.

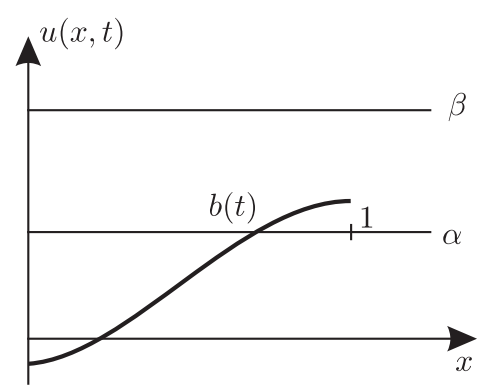

$t=t_{1}$

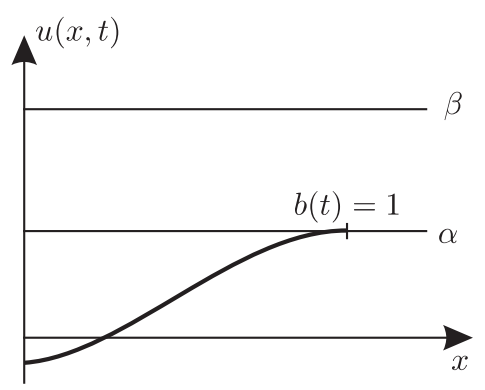

$t=T$

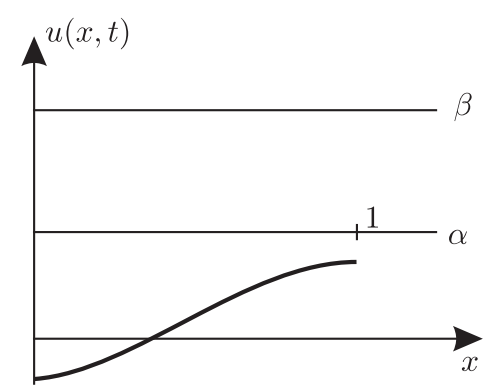

$t=t_{2}$

Figure 4.3: Transversality persists, but topology changes: $\mathcal{H}=1$ for $t \geq T$

\subsection{Continuous dependence of solutions on initial data}

In this subsection we prove an analogue of Theorem 2.3 under the assumptions from Sec. 2.5. The proof will consist of two steps. First, we shall prove the continuous dependence on small time intervals and then on the whole interval $[0, T]$. 


\subsubsection{Continuous dependence on small time interval}

Let the initial data $\varphi(x)$ and $\xi_{0}(x)$ satisfy Condition 2.8. By part 1 of Lemma 2.3, $\left(\varphi, \xi_{0}\right) \in E_{m}$ for some $m \in \mathbb{N}$. Let $\varphi_{n}(x)$ and $\xi_{0 n}(x)$ be other initial data satisfying Condition 2.8 Suppose that

$$
\left\|\varphi_{n}-\varphi\right\|_{W_{q, N}^{2-2 / q}} \rightarrow 0, \quad \bar{b}_{n}-\bar{b} \rightarrow 0, \quad n \rightarrow \infty,
$$

where $\bar{b}_{n}$ is the discontinuity point of $\xi_{0 n}(x)$.

By part 3 of Lemma 2.3, there is $n_{1}=n_{1}(m)>0$ such that

$$
\left(\varphi, \xi_{0}\right),\left(\varphi_{n}, \xi_{0 n}\right) \in E_{m+1} \quad \forall n \geq n_{1}(m) .
$$

In what follows, we assume $n \geq n_{1}(m)$. By Theorem 4.1, there is $T_{1}=T_{1}(m+1)>0$ for which problem (2.6)(2.8) has transverse topology preserving solutions $u, u_{n} \in W_{q}^{2,1}\left(Q_{T_{1}}\right)$ with the initial data $\varphi, \xi_{0}$ and $\varphi_{n}, \xi_{0 n}$, respectively. Moreover, any solution of problem (2.6)-(2.8) in $Q_{T_{1}}$ is transverse and preserves topology.

We introduce the functions $a(t)$ and $a_{n}(t)$ corresponding to $u$ and $u_{n}$ as described in part 3 of Theorem 3.2 . Then the discontinuity points of the corresponding configuration functions $\xi(x, t), \xi_{n}(x, t)$ are given by

$$
b(t)=\max _{s \in[0, t]} a(s), \quad b_{n}(t)=\max _{s \in[0, t]} a_{n}(s), \quad t \in\left[0, T_{1}\right] .
$$

Lemma 4.1. Under the above assumptions and the additional assumption that $u$ is a unique solution of problem (2.6)-(2.8) in $Q_{T_{1}}$ with the initial data $\varphi, \xi_{0}$, we have

$$
\left\|u_{n}-u\right\|_{W_{q}^{2,1}\left(Q_{T_{1}}\right)} \rightarrow 0, \quad\left\|b_{n}-b\right\|_{C\left[0, T_{1}\right]} \rightarrow 0, \quad n \rightarrow \infty .
$$

Proof. 1. Assume to the contrary that there is $\varepsilon>0$ such that

$$
\left\|u_{n}-u\right\|_{W_{q}^{2,1}\left(Q_{T_{1}}\right)} \geq \varepsilon, \quad n=1,2, \ldots,
$$

for some subsequence of $u_{n}$, which we denote $u_{n}$ again.

Theorem 4.1 implies that, for all sufficiently large $n$, the functions $u_{n}$ and $a_{n}$ are uniformly bounded in $W_{q}^{2,1}\left(Q_{T_{1}}\right)$ and $C^{\gamma}\left[0, T_{1}\right]$, respectively. Therefore, we can choose subsequences of $u_{n}$ and $a_{n}$ (which we denote $u_{n}$ and $a_{n}$ again) such that

$$
\begin{gathered}
\left\|u_{n}-\hat{u}\right\|_{C^{\gamma}\left(\bar{Q}_{T_{1}}\right)} \rightarrow 0,\left\|\left(u_{n}\right)_{x}-\hat{u}_{x}\right\|_{C^{\gamma}\left(\bar{Q}_{T_{1}}\right)} \rightarrow 0, \quad n \rightarrow \infty, \\
\left\|a_{n}-\hat{a}\right\|_{C\left[0, T_{1}\right]} \rightarrow 0, \quad n \rightarrow \infty
\end{gathered}
$$

for some function $\hat{u} \in C^{\gamma}\left(\bar{Q}_{T_{1}}\right)$ with $\hat{u}_{x} \in C^{\gamma}\left(\bar{Q}_{T_{1}}\right)$ and some function $\hat{a} \in C\left[0, T_{1}\right]$.

Denote

$$
\hat{b}(t)=\max _{s \in[0, t]} \hat{a}(s), \quad t \in\left[0, T_{1}\right] .
$$

It follows from (4.9), (4.10), and Lemma 2.4 that the functions $\hat{u}$ and $\hat{a}$ are such that

$$
\mathcal{H}(\xi(x), \hat{u}(x, \cdot))(t)= \begin{cases}H_{1}(\hat{u}(x, t)), & 0 \leq x \leq \hat{b}(t), \\ H_{2}(\hat{u}(x, t)), & \hat{b}(t)<x \leq 1\end{cases}
$$

Combining (4.10) with Lemma 3.3, we obtain

$$
\left\|b_{n}-\hat{b}\right\|_{C\left[0, T_{1}\right]} \rightarrow 0, \quad n \rightarrow \infty .
$$

2. Now using (4.5), (4.9), (4.11), and part 2 of Theorem 3.2, we see that the sequence of functions $u_{n}$ satisfying the problems

$$
\left\{\begin{array}{l}
\left(u_{n}\right)_{t}=\left(u_{n}\right)_{x x}+f\left(u_{n}, \mathcal{H}\left(\xi_{n}, u_{n}\right)\right), \quad(x, t) \in Q_{T_{1}}, \\
\left.\left(u_{n}\right)_{x}\right|_{x=0}=\left.\left(u_{n}\right)_{x}\right|_{x=1}=0, \\
\left.u_{n}\right|_{t=0}=\varphi_{n}(x), \quad x \in(0,1),
\end{array}\right.
$$


converges in $W_{q}^{2,1}\left(Q_{T_{1}}\right)$ to $\hat{u}$ satisfying the problem

$$
\left\{\begin{array}{l}
\hat{u}_{t}=\hat{u}_{x x}+f(\hat{u}, \mathcal{H}(\xi, \hat{u})), \quad(x, t) \in Q_{T_{1}}, \\
\left.\hat{u}_{x}\right|_{x=0}=\left.\hat{u}_{x}\right|_{x=1}=0 \\
\left.\hat{u}\right|_{t=0}=\varphi(x), \quad x \in(0,1) .
\end{array}\right.
$$

But the latter problem has a unique solution by assumption. Hence, $\hat{u}=u$, which contradicts (4.8).

Thus, the first convergence in the lemma is proved. The second convergence follows from (4.11).

\subsubsection{Continuous dependence on the whole interval without change of topology}

Now we shall prove Theorem 2.3 under the assumptions from Sec. 2.5. In particular, we assume that $\varphi$ and $\xi_{0}$ satisfy Condition 2.8. By assumption, problem (2.6)-(2.8) has a unique transverse topology preserving solution $u$ in $Q_{s}$ for any $s \leq T$. We denote by $b(t)$ the (unique) discontinuity point of the corresponding configuration function $\xi(x, t)$.

Further, we assume that $\varphi_{n}, \xi_{0 n}, n=1,2, \ldots$, is a sequence of other initial data satisfying Condition 2.8 such that

$$
\left\|\varphi_{n}-\varphi\right\|_{W_{q, N}^{2-2 / q}} \rightarrow 0, \quad \bar{b}_{n}-\bar{b} \rightarrow 0, \quad n \rightarrow \infty,
$$

where $\bar{b}_{n}$ is the discontinuity point of $\xi_{0 n}$.

We have to show that, for all sufficiently large $n$, problem (2.6)-(2.8) with the initial data $\varphi_{n}, \xi_{0 n}$ has at least one transverse topology preserving solution $u_{n} \in W_{q}^{2,1}\left(Q_{T}\right)$ and each sequence of such solutions satisfies

$$
\left\|u_{n}-u\right\|_{W_{q}^{2,1}\left(Q_{T}\right)} \rightarrow 0, \quad\left\|b_{n}-b\right\|_{C[0, T]} \rightarrow 0, \quad n \rightarrow \infty
$$

where $b_{n}(t)$ is the discontinuity point of $\xi_{n}(x, t)$.

Proof. 1. By Lemma 2.3, there is $m \in \mathbb{N}$ such that $(u(\cdot, t), \xi(\cdot, t)) \in E_{m}$ for all $t \in[0, T]$. Let us fix such a number $m$. Suppose that (4.13) does not hold. Let $\tau$ be the infimum of the set of all $s \in[0, T]$ such that at least one of the convergences

$$
\left\|u_{n}-u\right\|_{W_{q}^{2,1}\left(Q_{s}\right)} \rightarrow 0, \quad\left\|b_{n}-b\right\|_{C[0, s]} \rightarrow 0, \quad n \rightarrow \infty
$$

does not hold. By assumption $\tau<T$. On the other hand, Lemma 4.1 implies that $\tau \geq T_{1}$. In particular, this means that

$$
\left\|u_{n}\left(\cdot, \tau-T_{1} / 2\right)-u\left(\cdot, \tau-T_{1} / 2\right)\right\|_{W_{q, N}^{2-2 / q}} \rightarrow 0, \quad b_{n}\left(\tau-T_{1} / 2\right)-b\left(\tau-T_{1} / 2\right) \rightarrow 0, \quad n \rightarrow \infty .
$$

Applying Lemma 4.1 again, we obtain the convergence in (4.14) for $s=\min \left(\tau+T_{1} / 2, T\right)>\tau$. This contradiction proves (4.13).

\subsubsection{Continuous dependence on the whole interval with change of topology}

It remains to prove Corollary 2.1 under the assumptions from Sec. 2.5. In particular, we shall keep notations from the previous Sec. 4.3.2. However, we additionally assume that $t_{\max }>0$ is the number where the topology of $u$ changes, i.e.,

$$
b\left(t_{\max }\right)=1, \quad u\left(1, t_{\max }\right)=0,
$$

and that the solutions $u$ and $u_{n}$ are transverse on the interval $[0, T]$ with $T \geq t_{\max }$.

Lemma 4.2. $\left\|u-u_{n}\right\|_{W_{q}^{2,1}\left(Q_{t_{\max }}\right)} \rightarrow 0$ and $\left\|b-b_{n}\right\|_{C\left[0, t_{\max }\right]} \rightarrow 0$ as $n \rightarrow \infty$. 
Proof. 1. Let us prove the first convergence in the lemma. Assume to the contrary that there is $\varepsilon>0$ such that

$$
\left\|u-u_{n}\right\|_{W_{q}^{2,1}\left(Q_{t_{\max }}\right)} \geq \varepsilon, \quad n=1,2, \ldots,
$$

for some subsequence of $u_{n}$, which we denote $u_{n}$ again.

Theorem 2.3 implies that

$$
\left\|u-u_{n}\right\|_{W_{q}^{2,1}\left(Q_{t_{\max }-\delta}\right)} \rightarrow 0, \quad\left\|b-b_{n}\right\|_{C\left[0, t_{\max }-\delta\right]} \rightarrow 0, \quad n \rightarrow \infty
$$

for all (small) $\delta>0$. Therefore,

$$
\begin{gathered}
u_{n}(x, t) \rightarrow u(x, t) \quad \text { a.e. }(x, t) \in Q_{t_{\max }}, \\
b_{n}(t) \rightarrow b(t) \quad \text { a.e. } t \in\left(0, t_{\max }\right), \quad n \rightarrow \infty .
\end{gathered}
$$

Furthermore, Remark 3.2 and Lemma 3.2 imply that the functions $u_{n}$ are uniformly bounded in $W_{q}^{2,1}\left(Q_{t_{\text {max }}}\right)$. Therefore, we can choose a subsequence of $u_{n}$ (which we denote $u_{n}$ again) converging in $C\left(\bar{Q}_{t_{\text {max }}}\right)$. Taking into account (4.17), we see that it converges to $u$ :

$$
\left\|u-u_{n}\right\|_{C\left(\bar{Q}_{t_{\max }}\right)} \rightarrow 0, \quad n \rightarrow \infty
$$

On the other hand, it follows from (4.18), from the uniform boundedness of $b_{n}(t)$, and from Lebesgue's dominated convergence theorem that

$$
\left\|b-b_{n}\right\|_{L_{1}\left(0, t_{\max }\right)} \rightarrow 0, \quad n \rightarrow \infty
$$

Combining the convergence of initial data, relations (4.19) and (4.20), and part 2 of Theorem 3.2, we conclude that

$$
\left\|u-u_{n}\right\|_{W_{q}^{2,1}\left(Q_{t_{\max }}\right)} \rightarrow 0, \quad n \rightarrow \infty
$$

for the chosen subsequence. This contradicts (4.15). Therefore, (4.21) holds for the whole sequence $u_{n}$.

2. Now we prove the second convergence in the lemma. Suppose it does not hold. Then, due to the second convergence in (4.16), there is a subsequence of $t_{n}$ (which we denote $t_{n}$ again) and a number $\varepsilon>0$ such that $t_{n} \rightarrow t_{\max }$ and

$$
\left|b_{n}\left(t_{n}\right)-b\left(t_{n}\right)\right| \geq \varepsilon
$$

Combining this with the fact that $b\left(t_{n}\right) \rightarrow 1$, we see that there is a number $b^{*}<1$ such that

$$
b_{n}\left(t_{n}\right) \leq b^{*}
$$

On the other hand, since $b\left(t_{\max }\right)=1$, there is a moment $t^{*}<t_{\max }$ such that

$$
b\left(t^{*}\right)>b^{*}
$$

Now, using the monotonicity of $b_{n}$, the convergence in (4.16) and inequality (4.23), we have

$$
b_{n}\left(t_{n}\right) \geq b_{n}\left(t^{*}\right)>b^{*}
$$

for all sufficiently large $n$. This contradicts (4.22).

Proof of Corollary 2.1, Due to Lemma 4.2, it remains to show that

$$
\begin{gathered}
\left\|u_{n}-u\right\|_{W_{q}^{2,1}\left((0,1) \times\left(t_{\max }, T\right)\right)} \rightarrow 0, \quad n \rightarrow \infty, \\
\left\|b_{n}-b\right\|_{C\left[t_{\max }, T\right]} \rightarrow 0, \quad n \rightarrow \infty .
\end{gathered}
$$


1. Let us prove (4.24). Assume to the contrary that there is $\varepsilon>0$ such that

$$
\left\|u_{n}-u\right\|_{W_{q}^{2,1}\left((0,1) \times\left(t_{\max }, T\right)\right)} \geq \varepsilon, \quad n=1,2, \ldots,
$$

for some subsequence of $u_{n}$, which we denote $u_{n}$ again.

Remark 3.2 and Lemma 3.2 imply that the functions $u_{n}$ are uniformly bounded in $W_{q}^{2,1}\left(Q_{t_{\max }}\right)$. Therefore, there is a subsequence of $u_{n}$ (which we denote $u_{n}$ again) and a function $\hat{u}$ such that

$$
\left\|u_{n}-\hat{u}\right\|_{C\left([0,1] \times\left[t_{\max }, T\right]\right)} \rightarrow 0, \quad n \rightarrow \infty .
$$

Further, we have on the time interval $\left[t_{\max }, T\right]$ :

$$
\mathcal{H}\left(\xi_{0 n}, u_{n}\right)= \begin{cases}H_{1}\left(u_{n}\right), & 0 \leq x \leq b_{n}(t), \\ H_{2}\left(u_{n}\right), & b_{n}(t)<x \leq 1 .\end{cases}
$$

due to the transversality of $u_{n}$ (here we set $b_{n}(t)=1$ for $t>t_{n, \max }$ if $b_{n}\left(t_{n, \max }\right)=1$ ). Therefore, similarly to Lemma 3.4, we obtain

$$
\left\|\mathcal{H}\left(\xi_{0 n}, u_{n}\right)-H_{1}(\hat{u})\right\|_{L_{p}\left((0,1) \times\left(t_{\max }, T\right)\right)} \leq c_{0}\left(T-t_{\max }\right)^{1 / p}\left(\left\|\hat{u}-u_{n}\right\|_{C\left([0,1] \times\left[t_{\max }, T\right]\right)}^{\sigma}+\left|b_{n}\left(t_{\max }\right)-1\right|\right) .
$$

Together with Lemma 4.2 and relation (4.27), this yields

$$
\left.\| \mathcal{H}\left(\xi_{0 n}, u_{n}\right)-H_{1}(\hat{u})\right) \|_{L_{p}\left((0,1) \times\left(t_{\max }, T\right)\right)} \rightarrow 0, \quad n \rightarrow \infty .
$$

On the other hand, by Lemma 4.2 ,

$$
\left\|\left.u_{n}\right|_{t=t_{\max }}-\left.u\right|_{t=t_{\max }}\right\|_{W_{q, N}^{2-2 / q}} \rightarrow 0, \quad n \rightarrow \infty .
$$

Using (4.27)-4.29) and Theorem 3.1, we see that (cf. proof of part 2 of Theorem 3.2)

$$
\left\|u_{n}-\hat{u}\right\|_{W_{q}^{2,1}\left((0,1) \times\left(t_{\max }, T\right)\right)} \rightarrow 0, \quad n \rightarrow \infty,
$$

and $\hat{u}$ is a solution of the problem

$$
\left\{\begin{array}{l}
\left.\hat{u}_{t}=\hat{u}_{x x}+f\left(\hat{u}, H_{1}(\hat{u})\right), \quad(x, t) \in(0,1) \times\left(t_{\max }, T\right)\right), \\
\left.\hat{u}_{x}\right|_{x=0}=\left.\hat{u}_{x}\right|_{x=1}=0, \\
\left.\hat{u}\right|_{t=t_{\max }}=\left.u\right|_{t=t_{\max }}, \quad x \in(0,1) .
\end{array}\right.
$$

Due to the uniqueness assumption, $\hat{u}=u$ in $\left.(0,1) \times\left(t_{\max }, T\right)\right)$. Together with (4.30) this contradicts (4.26).

2. To prove (4.25), we note that, for all $t \in\left[t_{\max }, T\right]$,

$$
\left|b_{n}(t)-b(t)\right|=b\left(t_{\max }\right)-b_{n}(t) \leq b\left(t_{\max }\right)-b_{n}\left(t_{\max }\right) \rightarrow 0, \quad n \rightarrow \infty,
$$

due to Lemma 4.2

\section{Some generalizations}

In this section, we generalize Condition 2.4 to the following one (cf. Remark 2.3 and Example 2.1).

Condition 5.1 (generalized dissipativity). 1. For all sufficiently large $u$,

$$
f\left(u, H_{2}(u)\right) \leq 0, \quad f\left(-u, H_{1}(-u)\right) \geq 0
$$


2. there is a Lipschitz continuous function $h(u)$ such that $u h(u)>0$ for $u \neq 0$ and, for any $($ small $) \mu>0$, there exists $U_{\mu}>0$ such that the function

$$
f_{\mu}(u, v)=f(u, v)-\mu h(u)
$$

satisfies

$$
f_{\mu}\left(U_{\mu}, H_{j}(u)\right)<0, \quad f_{\mu}\left(-U_{\mu}, H_{j}(u)\right)>0 \quad \forall|u| \leq U_{\mu}, j=1,2 .
$$

Let us prove Theorem 2.1 under Condition 5.1 .

From now on we assume that $\left(\varphi, \xi_{0}\right) \in E_{m}$ for some $m$ (see Lemma 2.3) and $0<\mu \leq 1$. Due to part 2 of Condition [5.1 $f_{\mu}$ satisfies Condition 2.4. Therefore, by Theorems 2.1 and 2.2. problem (2.6) -(2.8) with the right-hand side $f_{\mu}$ has a solution $u_{\mu}$, which can be continued to a maximal interval of transverse existence $\left[0, T_{\mu, \max }\right)$.

Now the important step is to prove the boundedness of the solutions $u_{\mu}$ uniformly with respect to $\mu$.

Let us fix $U>\max (-\alpha, \beta)$ such that part 1 in Condition 5.1 holds for all $u \geq U$ and that $\|\varphi\|_{W_{q, N}^{2-2 / q}}<U$.

Lemma 5.1. The solutions $u_{\mu}$ satisfy $\left\|u_{\mu}\right\|_{C\left(\bar{Q}_{T_{\mu, m a x}}\right)}<U$.

Proof. Part 1 of Condition 5.1 and the assumption $u h(u)>0$ for $u \neq 0$ imply the strict inequalities

$$
f_{\mu}\left(U, H_{2}(U)\right)<0, \quad f_{\mu}\left(-U, H_{1}(-U)\right)>0 .
$$

Therefore, denoting $F(x, t)=f_{\mu}\left(u_{\mu}(x, t), \mathcal{H}\left(u_{\mu}(x, \cdot)\right)(t)\right)$, we can proceed analogously to the proof of Lemma 3.2 (with obvious modifications due to another definition of $F$ ).

Let $\left[0, t_{\mu, \max }\right)$ be a maximal interval, on which the solution $u_{\mu}$ both remains transverse and preserves spatial topology. We claim that there is $T>0$ such that $t_{\mu, \max } \geq T$ for all $\mu>0$. Indeed, suppose that there is a subsequence of $t_{\mu, \max }$ (which we denote $t_{\mu, \max }$ again) such that $t_{\mu, \max } \rightarrow 0$ as $\mu \rightarrow 0$. By Theorem 3.1 and Lemma 5.1 ,

$$
\begin{aligned}
\left\|u_{\mu}\right\|_{W_{q}^{2,1}\left(Q_{\left.t_{\mu, \max }\right)}+\right.}+\max _{t \in\left[0, t_{\mu, \max }\right]}\left\|u_{\mu}(\cdot, t)\right\|_{W_{q, N}^{2-2 / q}} \leq c_{1}\left(\|\varphi\|_{W_{q, N}^{2-2 / q}}+f_{U}+h_{U}\right), \\
\left\|u_{\mu}\right\|_{C^{\gamma}\left(\bar{Q}_{t_{\mu, \max }}\right)}+\left\|\left(u_{\mu}\right)_{x}\right\|_{C^{\gamma}\left(\bar{Q}_{t_{\mu, \max }}\right)} \leq c_{2}\left(\|\varphi\|_{W_{q, N}^{2-2 / q}}+f_{U}+h_{U}\right),
\end{aligned}
$$

where $f_{U}$ is defined in (3.10), $h_{U}=\max _{|u| \leq U}|h(u)|$, and $c_{2}>0$ does not depend on $\mu, t_{\mu, \max }, \varphi$.

The latter estimate shows that $u_{\mu}$ remains transverse and preserves spatial topology for all sufficiently small $t_{\mu, \max }$. This contradiction proves that $t_{\mu, \max } \geq T>0$.

Applying Theorem 3.1 and Lemma 5.1 again, we obtain the estimates

$$
\begin{gathered}
\left\|u_{\mu}\right\|_{C\left(\bar{Q}_{T}\right)}<U \\
\left\|u_{\mu}\right\|_{W_{q}^{2,1}\left(Q_{T}\right)}+\max _{t \in[0, T]}\left\|u_{\mu}(\cdot, t)\right\|_{W_{q, N}^{2-2 / q}} \leq c_{1}\left(\|\varphi\|_{W_{q, N}^{2-2 / q}}+f_{U}+h_{U}\right), \\
\left\|u_{\mu}\right\|_{C^{\gamma}\left(\bar{Q}_{T}\right)}+\left\|\left(u_{\mu}\right)_{x}\right\|_{C^{\gamma}\left(\bar{Q}_{T}\right)} \leq c_{2}\left(\|\varphi\|_{W_{q, N}^{2-2 / q}}+f_{U}+h_{U}\right),
\end{gathered}
$$

where $c_{1}, c_{2}>0$ do not depend on $\mu$.

For each $u_{\mu}$, we have a corresponding function $a_{\mu}(t)$ (cf. part 3 of Theorem 3.2). It follows from (5.2) and from the fact that $a_{\mu} \in C^{\gamma}[0, T]$ that one can choose subsequences, which we denote $u_{\mu}$ and $a_{\mu}$ again, converging to some functions $u(x, t)$ and $a(t)$ in $C(\bar{Q})$ and $C[0, T]$, respectively.

Using (5.2), one can show that the function $a(t)$ corresponds to $u(x, t)$ in the same sense as the functions $a_{\mu}(t)$ correspond to $u_{\mu}(x, t)$. In particular, this means that the corresponding hysteresis operators are given by

$$
\mathcal{H}\left(\xi_{0}, u\right)=\left\{\begin{array}{ll}
H_{1}(u), & 0 \leq x \leq b(t), \\
H_{2}(u), & b(t)<x \leq 1,
\end{array} \quad \mathcal{H}\left(\xi_{0}, u_{\mu}\right)= \begin{cases}H_{1}\left(u_{\mu}\right), & 0 \leq x \leq b_{\mu}(t), \\
H_{2}\left(u_{\mu}\right), & b_{\mu}(t)<x \leq 1,\end{cases}\right.
$$


where $b(t)=\max _{s \in[0, t]} a(s)$ and $b_{\mu}(t)=\max _{s \in[0, t]} a_{\mu}(s)$.

By Lemma 3.3. $\left\|b_{\mu}-b\right\|_{C[0, T]} \rightarrow 0$ as $\mu \rightarrow 0$. Thus, applying Remark 3.1 and Lemma 3.4, we see that $\mathcal{H}\left(\xi_{0}, u_{\mu}\right)$ converges to $\mathcal{H}\left(\xi_{0}, u\right)$ in $L_{q}\left(Q_{T}\right)$. Now the Lipschitz continuity of $f$ and the estimate $\left|h\left(u_{\mu}\right)\right| \leq h_{U}$ imply that $f_{\mu}\left(u_{\mu}, \mathcal{H}\left(\xi_{0}, u_{\mu}\right)\right)$ converges to $f\left(u, \mathcal{H}\left(\xi_{0}, u\right)\right)$ in $L_{q}\left(Q_{T}\right)$.

Denote by $\hat{u}$ the solution of the linear parabolic problem

$$
\begin{gathered}
\hat{u}_{t}=\hat{u}_{x x}+f\left(u, \mathcal{H}\left(\xi_{0}, u\right)\right), \quad(x, t) \in Q_{T}, \\
\left.\hat{u}_{x}\right|_{x=0}=\left.\hat{u}_{x}\right|_{x=1}=0, \\
\left.\hat{u}\right|_{t=0}=\varphi(x), \quad x \in(0,1) .
\end{gathered}
$$

By Theorem 3.1, $u_{\mu} \rightarrow \hat{u}$ in $W_{q}^{2,1}\left(Q_{T}\right)$, hence in $C\left(\bar{Q}_{T}\right)$. Therefore, $u=\hat{u}, u$ is a solution of problem (2.6)(2.8) with the right-hand side $f$, and estimates (5.1) and (5.2) yield the same estimates for $u$. Theorem 2.1 is proved.

The number $T$ which we have obtained above depends only on $m$. Therefore, the continuation theorem (Theorem 2.2) and the theorem on continuous dependence of solutions on initial data (Theorem 2.3) under Condition 5.1 are proved similarly to Secs. 4.2 and 4.3 (with the help of estimates (5.1) and (5.2), which now hold with $u$ ). The proof of Theorem 2.4 does not depend on the dissipativity condition at all (see 44).

Acknowledgement: The authors are grateful to Willi Jäger for drawing their attention to the field of hysteresis and to Bernold Fiedler and Alexander Nazarov for fruitful discussions. The research of the first author was supported by the DFG project SFB 910, by the DAAD program G-RISC, and by the RFBR (project 10-01-00395-a). The research of the third author was supported by the Alexander von Humboldt Foundation.

\section{References}

[1] H. W. Alt, On the thermostat problem. Control Cyb., 14, 171-193 (1985).

[2] A. Ashyralyev, P. E. Sobolevskii. Well-posedness of Parabolic Difference Equations. Operator Theory: Advances and Applications, 69. Birkhäuser Verlag, Basel (1994).

[3] L. C. Evans, M. Portilheiro, Irreversibility and hysteresis for a forward-backward diffusion equation. Math. Models Methods Appl. Sci., 14, no. 11, 1599-1620 (2004).

[4] P. Gurevich, S. Tikhomirov, Uniqueness of transverse solutions for reaction-diffusion equations with spatially distributed hysteresis. Preprint.

[5] F. C. Hoppensteadt, W. Jäger, Pattern formation by bacteria Lecture Notes in Biomathematics 38, 68-81 (1980).

[6] F. C. Hoppensteadt, W. Jäger, C. Poppe, A hysteresis model for bacterial growth patterns. Modelling of Patterns in Space and Time, Lecture Notes in Biomath. 55 (Springer), 123-134 (1984).

[7] A. M. Ilin, B. A. Markov, Nonlinear diffusion equation and Liesegang rings. Doklady Akademii Nauk, 440, No. 2, 164-167 (2011); English translation: Doklady Mathematics, 84, No. 2, 730-733 (2011).

[8] J. Kopfova, Hysteresis in biological models. Journal of Physics: Conference Series, 55, 130-134 (2006).

[9] M. A. Krasnosel'skii, A. V. Pokrovskii. Systems with Hysteresis. Springer-Verlag. Berlin-HeidelbergNew York (1989). Translated from Russian: Sistemy s Gisterezisom. Nauka. Moscow (1983).

[10] O. A. Ladyzhenskaya, V. A. Solonnikov, N. N. Uraltseva. Linear and Quasilinear Equations of Parabolic Type. Nauka, Moscow, 1967; English translation: Amer. Math. Soc., Providence, RI, 1968. 
[11] P. I. Plotnikov, Passing to the limit with respect to the viscosity in an equation with variable parabolicity direction, Differential Equations, 30, 614-622 (1994).

[12] F. Rothe. Global Solutions of Reaction-Diffusion Systems. Lecture Notes in Mathematics, 1072. SpringerVerlag, Berlin (1984).

[13] J. Smoller. Shock Waves and Reaction-Diffusion Equations. Second edition. Grundlehren der Mathematischen Wissenschaften [Fundamental Principles of Mathematical Sciences], 258. Springer-Verlag, New York (1994).

[14] H. Triebel. Interpolation Theory, Function Spaces, Differential Operators. Second edition. Johann Ambrosius Barth, Heidelberg (1995).

[15] A. Visintin Differential Models of Hysteresis. Springer-Verlag. Berlin - Heidelberg (1994).

[16] A. Visintin Evolution problems with hysteresis in the source term. SIAM J. Math. Anal, 17, 1113-1138 (1986). 